\title{
Relativistic warm plasma theory of nonlinear laser-driven electron plasma waves
}

\author{
C. B. Schroeder and E. Esarey \\ Lawrence Berkeley National Laboratory, Berkeley, California 94720 USA
}

(Dated: May 16, 2010)

\begin{abstract}
A relativistic, warm fluid model of a nonequilibrium, collisionless plasma is developed and applied to examine nonlinear Langmuir waves excited by relativistically-intense, short-pulse lasers. Closure of the covariant fluid theory is obtained via an asymptotic expansion assuming a non-relativistic plasma temperature. The momentum spread is calculated in the presence of an intense laser field and shown to be intrinsically anisotropic. Coupling between the transverse and longitudinal momentum variances is enabled by the laser field. A generalized dispersion relation is derived for langmuir waves in a thermal plasma in the presence of an intense laser field. Including thermal fluctuations in three velocity-space dimensions, the properties of the nonlinear electron plasma wave, such as the plasma temperature evolution and nonlinear wavelength, are examined, and the maximum amplitude of the nonlinear oscillation is derived. The presence of a relativistically intense laser pulse is shown to strongly influence the maximum plasma wave amplitude for non-relativistic phase velocities owing to the coupling between the longitudinal and transverse momentum variances.
\end{abstract}

\section{INTRODUCTION}

Short-pulse (sub-ps), intense $\left(\gtrsim 10^{18} \mathrm{~W} / \mathrm{cm}^{2}\right.$, such that the electron quiver velocity in the laser field is relativistic) laser-plasma interactions access a physical regime where the plasma electrons experience relativistic motion while the plasma temperature (momentum spread) remains small. The plasma is typically created by the laser through photoionization and the laser-plasma interaction occurs on a time-scale short compared to the ion motion and the collision frequency. Such a collisionless plasma is not in local thermodynamical equilibrium, where standard relativistic collisionally-dominated plasma fluid theories apply (see, for example, Ref. [1]). In this work, a relativistic warm plasma fluid theory of the nonequilibrium, collisionless plasma is used to describe the nonlinear plasma wave excitation by an intense laser pulse.

The electron plasma wave driven by an intense laser pulse can be highly-nonlinear and support large electric fields. Large amplitude plasma waves with relativistic phase velocities can be used to efficiently accelerate charged particles, and high-intensity lasers have been proposed for the excitation of relativistic plasma waves for plasma-based accelerator applications (for a review, see Ref. [2]). Laser-plasma-based accelerator experiments [3-6] have measured electric fields in excess of $100 \mathrm{GV} / \mathrm{m}$, several orders of magnitude greater then conventional accelerators. The relativistic warm fluid theory of the plasma described in this work can be applied to address the fundamental question of the maximum amplitude of these nonlinear relativistic electron plasma waves driven by intense lasers [7].

The subject of relativistic nonlinear oscillations in a plasma was first treated by Akhiezer and Polovin [8]. Using the nonlinear, relativistic, cold fluid equations in one-dimension (1D), Akhiezer and Polovin showed, assuming a traveling wave solution, that the maximum amplitude of a plasma wave that can sustain an oscillation in the plasma is $E_{\text {cold }}=\sqrt{2}\left(\gamma_{\varphi}-1\right)^{1 / 2} E_{0}$, which is re- ferred to as the cold relativistic wavebreaking field. Here $\gamma_{\varphi}=\left(1-\beta_{\varphi}^{2}\right)^{-1 / 2}$ is the relativistic Lorentz factor of the plasma wave phase velocity, and $E_{0}=c m \omega_{p} / e$ is referred to as the non-relativistic cold wavebreaking field, with $\omega_{p}=\left(4 \pi n_{0} e^{2} / m\right)^{1 / 2}=k_{p} c$ the plasma frequency, $n_{0}$ the ambient electron plasma number density, $-e$ the electronic charge, $m$ the rest mass of the electron, and $c$ the speed of light in vacuo. In the limit of non-relativistic phase velocities, $\beta_{\varphi} \ll 1, E_{\text {cold }} \simeq \beta_{\varphi} E_{0}$. For a plasma wave driven by the ponderomotive force of a short-pulse laser in an underdense plasma, the phase velocity of the plasma wave is approximately the laser group velocity, $\gamma_{\varphi} \simeq \omega_{0} / \omega_{p} \gg 1$ in the linear regime (assuming a broad laser spot size), where $\omega_{0}$ is the laser frequency. For plasma waves driven by Raman backscatter of the laser (i.e., generated by the beating of a laser and its backscattered light), the phase velocity of the plasma wave in the linear regime is non-relativistic $\beta_{\varphi} \approx \omega_{p} / 2 \omega_{0} \ll 1$.

The analysis of Akhiezer and Polovin [8] is based on a cold, collisionless fluid theory. When the amplitude of the plasma wave field approaches $E_{\text {cold }}=\sqrt{2}\left(\gamma_{\varphi}-1\right)^{1 / 2} E_{0}$, the cold plasma density becomes singular $n \rightarrow \infty$, at which point fluid oscillations can no longer be sustained. For a cold plasma, this singularity corresponds to a peak amplitude of the fluid velocity equal to the phase velocity of the wave, which implies a strong wave-particle resonance. This singularity is often referred to as "wavebreaking", and was first described by Dawson [9]. The singularity indicates a breakdown of the fluid equations. In this limit, the cold, collisionless fluid model becomes invalid and a warm plasma model is required.

Coffey [10] first considered large amplitude plasma waves including the effects of a finite plasma temperature and calculated, assuming a 1D waterbag momentum distribution of the plasma, the maximum field amplitude for a non-relativistic $\left(\gamma_{\varphi} \simeq 1\right)$ plasma wave,

$$
\frac{E_{\mathrm{C}}^{2}}{\beta_{\varphi}^{2} E_{0}^{2}} \simeq\left[1-\frac{8}{3}\left(\frac{3 \theta}{\beta_{\varphi}^{2}}\right)^{1 / 4}+2\left(\frac{3 \theta}{\beta_{\varphi}^{2}}\right)^{1 / 2}-\frac{1}{3}\left(\frac{3 \theta}{\beta_{\varphi}^{2}}\right)\right],
$$


where $\theta=k_{\mathrm{B}} \mathrm{T}_{e 0} / m c^{2}$ is the normalized initial electron plasma temperature with $k_{\mathrm{B}}$ the Boltzmann constant. Equation (1) is valid for $\theta^{1 / 2} \ll \beta_{\varphi} \ll 1$ and indicates that temperature effects reduce the maximum field amplitude of the plasma wave.

A plasma wave driven by a short-pulse (pulse duration on the order of the plasma period) laser will have a phase velocity approximately equal to the group velocity of the laser pulse, typically $\gamma_{\varphi} \approx \omega_{0} / \omega_{p} \sim 10-100$ for laser propagation in an underdense plasma (e.g., $n_{0} \sim 10^{17}$ $10^{19} \mathrm{~cm}^{-3}$ and $\left.\lambda_{0}=2 \pi c / \omega_{0} \sim 1 \mu \mathrm{m}\right)$. Without some additional heating mechanism, laboratory plasmas used for short-pulse laser-plasma experiments have temperatures of the order of $\theta m c^{2} \sim 10 \mathrm{eV}$ (i.e., the ionization potential) [11, 12]. Therefore, a short-pulse laser-driven plasma wave (e.g., a laser-driven plasma-based accelerator) will satisfy $\theta^{1 / 2}<\gamma_{\varphi}^{-1} \ll 1$. In Ref. [7], a general result for the maximum field of a nonlinear electron plasma wave was presented, which can be applied to the regime of laser-driven plasma waves and reduces to the previous wavebreaking calculations in the cold and non-relativistic limits [8-10]. This general 1D wavebreaking result was derived using the relativistic warm plasma theory presented in this work. Warm 1D fluid theories $[13,14]$ have also been applied in the limit of ultra-relativistic plasma waves $\beta_{\varphi}=1$, which are valid for plasma waves driven by highly-relativistic electron beams satisfying $\gamma_{\varphi}^{2} \theta \gg 1$, and discrepancies between previous 1D ultra-relativistic theories $[13,14]$ have been discussed in Ref. [15].

In this paper we extend the relativistic fluid theory used in Ref. [7] to include the transverse thermal fluctuations. A warm plasma assumption (i.e., the distribution has a small width about a relativistic mean motion) allows closure of the hierarchy of equations for the moments of the phase space distribution. The evolution of the momentum variance is derived in the presence of an intense laser field. The laser field is assumed to be a plane wave. Here we show that the momentum spread is highly anisotropic. This is in agreement with the Hamiltonian description found in Refs. [16, 17], and in contrast to the findings of Ref. [18] that predicted isotropic pressure.

The general problem of three-dimensional (3D) adiabatic closure for a collisionless plasma is addressed in this work. Note that if the electron plasma is collisionless, i.e., $\nu_{c} \ll \omega_{p}$, where $\nu_{c}$ is the collision frequency, then changes in temperature (momentum spread) during the compression along the direction of wave propagation will not couple to the other two velocity dimensions, and the pressure will be anisotropic. In particular, the $3 \mathrm{D}$ adiabatic equation of state $p n^{-5 / 3}=$ constant, where $p$ is the isotropic pressure and $n$ is the plasma density, can not be applied to a Langmuir wave. In deriving $p n^{-5 / 3}=$ constant, an isotropic pressure is assumed (as well as no heat flow), which requires collisions to transfer energy among the dimensions faster than the wave period $\nu_{c} \gg \omega \sim \omega_{p}$. The collision frequency is $\nu_{c} / \omega_{p} \sim 1 / \Lambda$ [19], where $\Lambda$ is the plasma parameter, and the condition for a plasma requires $\Lambda \gg 1$. Hence, $\nu_{c} \ll \omega$ and the pressure for a Langmuir wave is intrinsically anisotropic. We show in this work that the presence of an intense laser pulse allows coupling between the anisotropic momentum variance components, and using the relativistic warm fluid equations a generalized Langmuir dispersion relation is derived including the influence of an intense laser field on the thermal fluctuations.

This paper is organized as follows. Section II describes a covariant fluid model of the collisionless plasma. Section III discusses the warm plasma assumption that allows closure of the fluid equations. The evolution for the anisotropic momentum variance is derived. From the linearized relativistic warm fluid equations, a generalized Langmuir dispersion relation is derived including the presence of an intense laser field. The quasi-static warm plasma response, assuming a travelling wave solution, is described in Sec. IV. In Sec. V, the laser excitation of nonlinear plasma waves is discussed, and a generalized quasi-static wave equation is derived including the transverse thermal fluctuations. From the quasi-static wave equation, the maximum field amplitude of the plasma wave (wavebreaking limit) is calculated. Conclusions are offered in Sec. VI.

\section{RELATIVISTIC FLUID EQUATIONS}

The Vlasov (collisionless Boltzmann) equation [1] for particles of charge $q$ and mass $m$, in covariant form, is

$$
p^{\mu} \partial_{\mu} f+\left(\frac{q}{m c^{2}} F^{\alpha \nu} p_{\nu}\right) \frac{\partial f}{\partial p^{\alpha}}=0,
$$

where $f\left(x^{\mu}, p^{\mu}\right)$ is the phase-space distribution (a Lorentz scalar $), x^{\mu}=(c t, \boldsymbol{x}), p^{\nu}=(\gamma, \gamma \boldsymbol{\beta})$ is the normalized particle 4-momentum, $\gamma^{2}=1+p^{2}, \partial^{\mu}=\left(\partial_{c t},-\nabla\right)$, and $F^{\mu \nu}=\partial^{\mu} A^{\nu}-\partial^{\nu} A^{\mu}$ is the antisymmetric electromagnetic field-strength tensor [20], with $A^{\mu}=(\Phi, \boldsymbol{A})$ the 4 -vector potential. The space-time metric tensor $g^{\mu \nu}$ for the following covariant formulation has the convention $g^{\mu \nu}=\operatorname{diag}(1,-1,-1,-1)$.

Following previous relativistic fluid formulations [2124], consider the following moments of the phase-space distribution:

$$
\begin{aligned}
h & =\int d \Omega f, \\
J^{\mu} & =\int d \Omega f p^{\mu}, \\
T^{\mu \nu} & =\int d \Omega f p^{\mu} p^{\nu}, \\
M^{\alpha \mu \nu} & =\int d \Omega f p^{\alpha} p^{\mu} p^{\nu},
\end{aligned}
$$

where $d \Omega=d^{3} \boldsymbol{p} / p^{0}=d p^{1} d p^{2} d p^{3} / p^{0}$ is the invariant momentum space volume element. Equations (3)-(6) correspond to the invariant density, fluid 4-current, energymomentum stress tensor, and energy-momentum stress 
flux, respectively. The inhomogeneous Maxwell equations can be expressed as

$$
\partial_{\mu} F^{\mu \nu}=4 \pi \sum_{s} q_{s} J_{s}^{\nu}
$$

where the sum is over species. The proper density may be defined using the 4-current as $n_{p}=\left(J^{\mu} J_{\mu}\right)^{1 / 2}$. Using Eq. (2), the fluid description of the plasma (i.e., the evolution of the moments of the phase-space distribution) can be written in terms of the exact collisionless conservation laws:

$$
\begin{aligned}
\partial_{\mu} J^{\mu} & =0 \\
\partial_{\mu} T^{\mu \nu} & =\left(\frac{q}{m c^{2}}\right) F^{\nu \sigma} J_{\sigma}, \\
\partial_{\alpha} M^{\alpha \mu \nu} & =\left(\frac{q}{m c^{2}}\right)\left(F^{\mu \sigma} T_{\sigma}^{\nu}+F^{\nu \sigma} T_{\sigma}^{\mu}\right) .
\end{aligned}
$$

Equations (8)-(10) correspond to the fluid continuity (mass conservation), energy-momentum conservation, and conservation of energy-momentum flux, respectively.

Using the fluid 4-momentum, defined as the ratio of the 4 -current to the invariant density,

$$
u^{\mu}=J^{\mu} / h .
$$

the following centered moments of the phase-space distribution may be defined as

$$
\begin{aligned}
\Theta^{\mu \nu} & =\int d \Omega f\left(p^{\mu}-u^{\mu}\right)\left(p^{\nu}-u^{\nu}\right), \\
Q^{\alpha \mu \nu} & =\int d \Omega f\left(p^{\alpha}-u^{\alpha}\right)\left(p^{\mu}-u^{\mu}\right)\left(p^{\nu}-u^{\nu}\right) .
\end{aligned}
$$

Note that there is freedom in choosing a fluid 4momentum and various definitions may be employed (see, for example, Refs. [1, 16]). The fluid 4-momentum Eq. (11) is related to the more standard choice of Eckart [25] $U^{\mu}=J^{\mu} /\left(J_{\mu} J^{\mu}\right)^{1 / 2}$ by a Lorentz invariant scalar, namely the ratio of proper to invariant densities: $u^{\mu}=$ $\left(n_{p} / h\right) U^{\mu}$.

The moments Eqs. (5) and (6) may be expressed using the centered moments Eqs. (12) and (13) as

$$
\begin{aligned}
T^{\mu \nu} & =h u^{\mu} u^{\nu}+\Theta^{\mu \nu}, \\
M^{\alpha \mu \nu} & =h u^{\alpha} u^{\mu} u^{\nu}+Q^{\alpha \mu \nu}+\Theta^{\mu \nu} u^{\alpha}+\Theta^{\mu \alpha} u^{\nu}+\Theta^{\alpha \nu} u^{\mu} .
\end{aligned}
$$

Note that the contractions of the moments satisfy $g_{\mu \nu} T^{\mu \nu}=h$ and $g_{\mu \nu} M^{\alpha \mu \nu}=J^{\alpha}$, and the contractions of the centered moments satisfy

$$
\begin{aligned}
g_{\mu \nu} \Theta^{\mu \nu} & =\left(1-u_{\mu} u^{\mu}\right) h, \\
g_{\mu \nu} Q^{\alpha \mu \nu} & =-2 \Theta^{\mu \alpha} u_{\mu} .
\end{aligned}
$$

The conservation laws Eqs. (8)-(10) may be expressed using the centered moments as the fluid equations [2124]:

$$
\begin{gathered}
\partial_{\mu}\left(h u^{\mu}\right)=0 \\
h u^{\mu} \partial_{\mu} u^{\nu}=-\partial_{\mu} \Theta^{\mu \nu}+\left(\frac{q}{m c^{2}}\right) F^{\nu \alpha} h u_{\alpha},
\end{gathered}
$$

and

$$
\begin{aligned}
& h u^{\alpha} \partial_{\alpha}\left(\Theta^{\mu \nu} / h\right)+\Theta^{\mu \alpha} \partial_{\alpha} u^{\nu}+\Theta^{\nu \alpha} \partial_{\alpha} u^{\mu} \\
& \quad=-\partial_{\alpha} Q^{\alpha \mu \nu}+\left(\frac{q}{m c^{2}}\right)\left(F^{\mu \alpha} \Theta_{\alpha}^{\nu}+F^{\nu \alpha} \Theta_{\alpha}^{\mu}\right) .
\end{aligned}
$$

The above fluid formulation is an exact consequence of the Vlasov equation Eq. (2) and no assumptions were made on the derivations of the above relations (the relativistic moment equations up to the fourth moment). In Appendix A the relativistic fluid quantities described in this section are related to conventional fluid quantities. In Sec. III a "warm" plasma assumption is described, where it is assumed that the distribution $f$ has a small width about a relativistic mean momentum. In particular, the third and higher-order centered moments (i.e., $Q^{\alpha \mu \nu}$ ) are assumed to be small and may be neglected, providing closure to the hierarchy of moment equations.

\section{WARM PLASMA ASSUMPTION}

In the following it is assumed that at any $x^{\mu}$, the distribution $f$ has a small momentum spread about its mean $[16,21-24]$. This warm plasma assumption will allow the hierarchy of moment equations to be truncated, and no specific form of the distribution $f$ will be imposed.

An invariant measure of thermal spread may be defined as $[21-24]$

$$
\epsilon^{2}=-\Theta_{\mu}^{\mu} / h=u^{\mu} u_{\mu}-1,
$$

where $\epsilon^{2} \ll 1$ is assumed to be small. Note that $\epsilon$ is a Lorentz invariant and allows a truncation valid in the rest frame to be preserved after an arbitrary Lorentz boost. In the local plasma rest frame (i.e., moving at $u^{\mu}$ ), it is assumed that $\Theta^{\mu \nu} / h=\mathcal{O}\left(\epsilon^{2}\right)$ and $Q^{\alpha \mu \nu} / h=\mathcal{O}\left(\epsilon^{3}\right)$, and the components satisfy $\Theta^{i j} / h=\mathcal{O}\left(\epsilon^{2}\right), \Theta^{i 0} / h=$ $\mathcal{O}\left(\epsilon^{3}\right), \Theta^{00} / h=\mathcal{O}\left(\epsilon^{4}\right)$, and $Q^{i j k} / h=\mathcal{O}\left(\epsilon^{3}\right), Q^{i j 0} / h=$ $\mathcal{O}\left(\epsilon^{4}\right), Q^{i 00} / h=\mathcal{O}\left(\epsilon^{5}\right), Q^{000} / h=\mathcal{O}\left(\epsilon^{6}\right)$. Truncation of the moment hierarchy to order $\mathcal{O}\left(\epsilon^{2}\right)$ is achieved by neglecting the third-order centered moment $Q^{\alpha \mu \nu}$ in the fluid equations [cf. Eq. (20)]. The invariant measure of thermal spread is related to the width of the distribution (i.e., temperature normalized to the electron rest energy). In Appendix B, the invariant measure $\epsilon$ is calculated for a relativistic Maxwellian (Jüttner) distribution and shown to be equal to the width of the momentum distribution.

As a consequence of the thermal inertia, the invariant density $h$ is not equal to the proper density defined by the 4-current $n_{p}=\left(J^{\mu} J_{\mu}\right)^{1 / 2}$. Following Refs. [21, 23], consider the ratio $\gamma_{\mathrm{th}}=n_{p} / h$. Note that $u^{\mu} u_{\mu}=\gamma_{\mathrm{th}}^{2}$. Using the contraction of the stress energy tensor $T_{\mu}^{\mu}=h$,

$$
\gamma_{\text {th }}^{2}=1-\Theta_{\mu}^{\mu} / h=1+\epsilon^{2},
$$

and $\gamma_{\text {th }}$ can be identified as the relativistic Lorentz factor associated with the thermal fluctuations. The fluid 3 -velocity normalized to the speed of light $\boldsymbol{w}$ may be 
defined such that $u^{\mu}=J^{\mu} / h=\gamma_{\mathrm{th}} \Gamma(1, \boldsymbol{w})$, where $\Gamma=(1-\boldsymbol{w} \cdot \boldsymbol{w})^{-1 / 2}$ is the Lorentz factor associated with the normalized fluid 3 -velocity. The invariant density $h$ is related to the density in the lab-frame $J^{0}=n$ by $h=n /\left(\gamma_{\mathrm{th}} \Gamma\right)$.

Neglecting the third-order centered moment $Q^{\alpha \mu \nu}$ [i.e., retaining terms to order $\left.\mathcal{O}\left(\epsilon^{2}\right)\right]$, the contraction Eq. (17) becomes

$$
\Theta^{\mu \nu} u_{\mu}=0
$$

and the fluid equations Eqs. (18), (19), and (20) become [to order $\left.\mathcal{O}\left(\epsilon^{2}\right)\right]$

$$
\begin{aligned}
u^{\mu} \partial_{\mu} h= & -h \partial_{\mu} u^{\mu} \\
h u^{\mu} \partial_{\mu} u^{\nu}= & -\partial_{\mu} \Theta^{\mu \nu}+\left(q / m c^{2}\right) F^{\nu \alpha} h u_{\alpha}, \\
h u^{\alpha} \partial_{\alpha}\left(\Theta^{\mu \nu} / h\right)= & -\Theta^{\mu \alpha} \partial_{\alpha} u^{\nu}-\Theta^{\nu \alpha} \partial_{\alpha} u^{\mu} \\
& +\left(q / m c^{2}\right)\left(F^{\nu \alpha} \Theta_{\alpha}^{\mu}+F^{\mu \alpha} \Theta_{\alpha}^{\nu}\right) .
\end{aligned}
$$

Equations (23)-(26) constitute a closed set of fluid equations, which may be solved for the plasma evolution.

\section{A. Warm-plasma closure for one-dimensional fluid motion}

In the limit of $1 \mathrm{D}$ fluid motion, i.e., $u^{\mu}=\gamma_{\mathrm{th}} \Gamma\left(1, w_{z}\right)$, Eq. (23) implies $\Theta^{00}=w_{z}^{2} \Theta^{11}$ and $\Theta^{10}=w_{z} \Theta^{11}$. Combining the evolution equation for the momentum-density variance $\Theta^{11}$, Eq. (26), the continuity equation Eq. (24), and the zeroth-component of the momentum equation Eq. (25), yields [to order $\mathcal{O}\left(\epsilon^{2}\right)$,

$$
u^{\mu} \partial_{\mu}\left(h^{-3} \Gamma^{-2} \Theta^{11}\right)=0
$$

where $\left(u^{0}\right)^{-1} u^{\mu} \partial_{\mu}=\left(\partial_{0}+w_{z} \partial_{1}\right)$ is the (noncovariant) convective time derivative. Equation (27) is a result of entropy conservation. Assuming an initially quiescent $\left(w_{z}=0\right)$, isotropic plasma, Eq. (27) implies

$$
\frac{\Theta^{11}}{n_{0}}=\Gamma^{2}\left(\frac{h}{n_{0}}\right)^{3} \theta
$$

where $\theta$ is the initial momentum variance (initial plasma temperature normalized to $\left.m c^{2} / k_{\mathrm{B}}\right)$. In the limit of $1 \mathrm{D}$ fluid motion, to order $\mathcal{O}\left(\epsilon^{2}\right)$, the invariant measure of thermal spread is given by

$$
\epsilon^{2}=\frac{n_{0}}{h}\left(\frac{\Theta^{11}}{n_{0}}-\frac{\Theta^{00}}{n_{0}}\right)=\left(\frac{h}{n_{0}}\right)^{2} \theta .
$$

The variance in momentum about the mean is given by $\Theta^{11} / h=\Gamma^{2} \epsilon^{2} \simeq\left(n / n_{0}\right)^{2} \theta$, and the root mean square variance in the velocity $\beta_{\text {th }}$ about the mean velocity $w_{z}$ is $\beta_{\mathrm{th}}=\epsilon / \Gamma^{2}$.

Equation (28) provides closure to the warm fluid equations in the limit of $1 \mathrm{D}$ fluid motion, and the momentum variance is proportional to the density cubed. This is sometimes referred to as the "adiabatic equation of state" [26]. Note that, the derivation of Eqs. (28) and (29) does not assume a specific form of the phase-space distribution $f$, or an equation of state (although only reversible processes are considered in this collisionless formulation). The derivation is a consequence of the asymptotic expansion (warm plasma approximation). Equation (28) is valid for any distribution $f$ provided $\epsilon^{2}=\theta\left(h / n_{0}\right)^{2} \ll 1$ (i.e., a warm plasma), and the plasma is initially quiescent.

The closed system of warm fluid equations in the limit of 1D fluid motion, coupled to the Poisson equation for the electrostatic field $\partial_{z}^{2} \phi=k_{p}^{2}\left(n / n_{0}-1\right)$, may be expressed as $\partial_{c t} n+\partial_{z}\left(n w_{z}\right)=0$ and

$$
\left(\partial_{c t}+w_{z} \partial_{z}\right) u_{z}=\partial_{z} \phi-\left[\partial_{z}\left(\Gamma^{2} \mathrm{p}\right)-\partial_{c t}\left(w_{z} \Gamma^{2} \mathrm{p}\right)\right] / n,
$$

where $n=J^{0}$ and the normalized 1D hydrostatic pressure may be defined as $\mathrm{p}=h \epsilon^{2}=\left(n / n_{0} \Gamma\right)^{3} n_{0} \theta$ (cf. Appendix A). Note that $u_{z}=u_{0} w_{z}$, where $u_{0}=\gamma_{\mathrm{th}} \Gamma=$ $\left(1+\epsilon^{2}\right)^{1 / 2}\left(1-w_{z}^{2}\right)^{-1 / 2}=\left(1+\epsilon^{2}+u_{z}^{2}\right)^{1 / 2}$ is the total relativistic Lorentz factor of the warm fluid, and $\epsilon^{2} \ll 1$ is assumed in the warm fluid model. Compared to the $1 \mathrm{D}$ relativistic cold fluid model, thermal effects are manifested in the pressure terms on the right hand side of Eq. (30), as well as the thermal correction to the relativistic Lorentz factor.

\section{B. Warm relativistic plasma in a laser field}

Consider a linearly-polarized drive laser (assumed to be a plane wave) propagating in the $z$-direction with transverse normalized vector potential $a_{\perp}=e A_{\perp} / m c^{2}$ (in the Coulomb gauge). Consider $1 \mathrm{D}$ spatial and $3 \mathrm{D}$ momentum-space motion of the plasma in the laser field such that the phase-space distribution is $f=$ $f\left(z, p^{\mu}, t\right)$, the fluid momentum takes the form $u^{\mu}=$ $\gamma_{\mathrm{th}} \Gamma\left(1, w_{z}, w_{\perp}, w_{3}\right)$ (with $w_{z}$ along the direction of laser propagation, $w_{\perp}$ along the polarization direction of the laser, and $w_{3}$ orthogonal to the laser polarization and propagation directions), and $\partial_{\mu}=\left(\partial_{0}, \partial_{1}\right)=\left(\partial_{c t}, \partial_{z}\right)$ (i.e., no transverse spatial variation).

For a plane wave laser pulse with normalized vector potential $a_{\perp}=e A_{\perp} / m c^{2}$ and space charge oscillation with normalized potential $\phi=e \Phi / m c^{2}$ (in the Coulomb gauge), the non-zero field strength tensor components are

$$
\begin{aligned}
& F^{10}=-F^{01}=-\left(m c^{2} / e\right) \partial_{1} \phi, \\
& F^{20}=-F^{02}=-\left(m c^{2} / e\right) \partial_{0} a_{\perp}, \\
& F^{12}=-F^{21}=-\left(m c^{2} / e\right) \partial_{1} a_{\perp} .
\end{aligned}
$$

In this work we consider an underdense plasma. For an underdense plasma a time scale separation exists between the fast laser oscillation (with time scale $\omega_{0}^{-1}$ ) and the slow plasma response (with time scale $\omega_{p}^{-1}$ ) such that $\omega_{p} / \omega_{0} \ll 1$. For the case of linear laser polarization, a time average over the fast laser oscillation may be performed such that $\left\langle a_{\perp}^{2}\right\rangle \simeq a_{0}^{2} / 2$, with 
$a_{0}^{2} \simeq 7.3 \times 10^{-19}(\lambda[\mu \mathrm{m}])^{2} I_{0}\left[\mathrm{~W} / \mathrm{cm}^{2}\right]$ and $I_{0}$ is the peak laser intensity. The plane wave assumption will be valid provided $k_{p} r_{L} \gg 1$ and $a_{0} \ll\left(k_{p} r_{L}\right)^{2}$, where $r_{L}$ is the characteristic transverse size of the laser.

The fluid equations can be combined to yield the components of the momentum variance Eq. (26) [to order $\mathcal{O}\left(\epsilon^{2}\right)$ ] assuming an initially quiescent plasma: $u^{\mu} \partial_{\mu}\left(\Theta^{33} / h\right)=0, u^{\mu} \partial_{\mu}\left(\Theta^{23} / h\right)=0, u^{\mu} \partial_{\mu}\left(\Theta^{22} / h\right)=0$,

$$
\begin{aligned}
u^{\mu} \partial_{\mu}\left(\frac{\Theta^{13}}{\Gamma h^{2}}\right) & =-\left(\frac{\Theta^{23}}{h^{2}}\right) \partial_{1}\left(a_{\perp} / \Gamma\right), \\
u^{\mu} \partial_{\mu}\left(\frac{\Theta^{12}}{\Gamma h^{2}}\right) & =-\left(\frac{\Theta^{22}}{h^{2}}\right) \partial_{1}\left(a_{\perp} / \Gamma\right), \\
u^{\mu} \partial_{\mu}\left(\frac{\Theta^{11}}{\Gamma^{2} h^{3}}\right) & =-2\left(\frac{\Theta^{12}}{\Gamma h^{3}}\right) \partial_{1}\left(a_{\perp} / \Gamma\right) .
\end{aligned}
$$

Equation (23) implies $\Theta^{\mu 0}=w_{z} \Theta^{\mu 1}+w_{\perp} \Theta^{\mu 2}+w_{3} \Theta^{\mu 3}$, and the additional components are

$$
\begin{aligned}
& \Theta^{00}=w_{z}^{2} \Theta^{11}+2 w_{z} w_{\perp} \Theta^{12}+w_{\perp}^{2} \Theta^{22}, \\
& \Theta^{01}=w_{z} \Theta^{11}+w_{\perp} \Theta^{12}, \\
& \Theta^{02}=w_{z} \Theta^{12}+w_{\perp} \Theta^{22} \\
& \Theta^{03}=w_{z} \Theta^{13}+w_{\perp} \Theta^{23} .
\end{aligned}
$$

The momentum variance tensor $\left(\Theta^{\mu \nu} / h\right)$ is anisotropic and Eqs. (34)-(36) indicate that the laser field allows coupling between the momentum variance components.

The momentum variance components can be combined to yield the invariant

$$
u^{\mu} \partial_{\mu}\left\{\left(\frac{\Theta^{33}}{h}\right)\left[\left(\frac{\Theta^{22}}{h}\right)\left(\frac{\Theta^{11}}{\Gamma^{2} h^{3}}\right)-\left(\frac{\Theta^{12}}{\Gamma h^{2}}\right)^{2}\right]\right\}=0,
$$

which is a result of entropy conservation. For an initially isotropic, quiescent plasma,

$$
\left(\frac{\Theta^{33}}{n_{0} \theta}\right)\left[\left(\frac{\Theta^{22}}{n_{0} \theta}\right)\left(\frac{\Theta^{11}}{n_{0} \theta}\right)-\left(\frac{\Theta^{12}}{n_{0} \theta}\right)^{2}\right]=\Gamma^{2}\left(\frac{h}{n_{0}}\right)^{5} .
$$

Equations (41) and (42) are 3D generalizations of the 1D results Eqs. (27) and (28), respectively [i.e., generalization of the 1D "adiabatic equation of state"].

\section{Dispersion relation for a electron plasma wave in an intense laser field}

Assuming a warm plasma in a laser field (closure given in Sec. IIIB), and applying the Fourier transformation $\left(\partial_{z}, \partial_{t}\right) \rightarrow(i k,-i \omega)$ to the linearized relativistic warm fluid equations [i.e., linearization of Eqs. (23)-(26)], coupled to the Poisson equation, yields the Langmuir wave

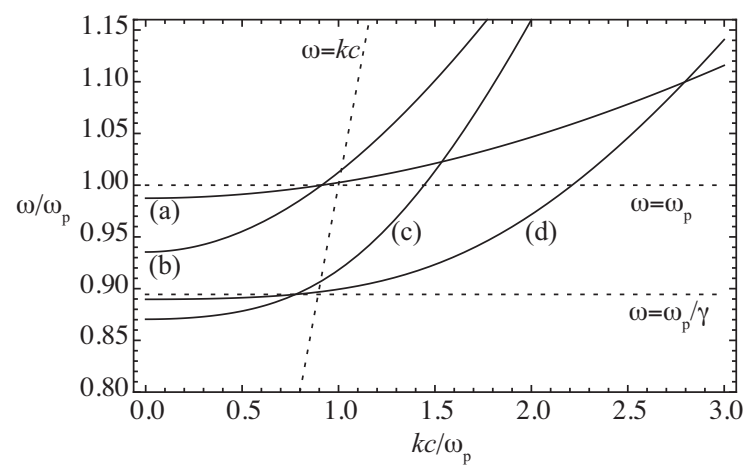

FIG. 1. Langmuir wave dispersion curves Eq. (43): (a) $\theta=$ $0.01 ; a=0$ (b) $\theta=0.05, a=0$; (c) $\theta=0.01, a=0.75$; and (d) $\theta=0.05, a=0.75$.

dispersion relation:

$$
\begin{aligned}
\omega^{2}=\frac{\omega_{p}^{2}}{\gamma_{\perp}}(1 & \left.+\frac{3}{2} \frac{a^{2} \theta}{\gamma_{\perp}^{2}}-\frac{5}{2} \frac{\theta}{\gamma_{\perp}^{2}}\right) \\
& +3 k^{2} c^{2} \frac{\theta}{\gamma_{\perp}^{2}}\left(1-\frac{3}{2} \frac{a^{2}}{\gamma_{\perp}^{2}}+\frac{a^{2} k^{2} c^{2}}{\gamma_{\perp}^{2} \omega^{2}}\right) .
\end{aligned}
$$

where $\theta$ is the initial isotropic plasma temperature (invariant measure of thermal spread), $\gamma_{\perp}^{2}=1+a^{2}$ is the relativistic Lorentz factor associated with the quiver motion in the laser field, and $a^{2}$ is assumed constant. For linear laser polarization, $a^{2} \rightarrow a_{0}^{2} / 2$, where $a_{0}$ is the normalized peak of the laser intensity. Figure 1 shows the Langmuir wave dispersion curves Eq. (43): Curves (a) $\theta=0.01$ and (b) $\theta=0.05$ show the effect of temperature on the Langmuir dispersion without a laser field (i.e., the relativistic Bohm-Gross dispersion). Curves (c) $\theta=0.01$ and (d) $\theta=0.05$ include the presence of a laser field with $a=0.75$. Also plotted are the speed of light line $\omega=k c$, the plasma frequency $\omega=\omega_{p}$, and the relativistic plasma frequency $\omega=\omega_{p} / \gamma_{\perp}$ for $a=0.75$. The phase velocity $\beta_{\varphi}=\omega / c k$ is strongly modified by the presence of an intense laser field in the limit of slow phase velocities (i.e., $k \gg k_{p}$ ). Without a laser field the phase velocity is bounded by $\beta_{\varphi}>(3 \theta)^{1 / 2}$. With an intense laser field (such that $a / \gamma_{\perp} \gg \beta_{\varphi}$ ), the phase velocity is bounded by $\omega / c k>(3 \theta)^{1 / 4} a^{1 / 2} / \gamma_{\perp}$.

The group velocity of plasma wave is

$$
\beta_{g}=\frac{\partial \omega}{c \partial k}=\frac{3 \theta}{\beta_{\varphi} \gamma_{\perp}^{2}}\left[1+\left(\frac{2}{\beta_{\varphi}^{2}}-\frac{3}{2}\right) \frac{a^{2}}{\gamma_{\perp}^{2}}\right]
$$

In the absence of a laser field, $\beta_{g} \beta_{\varphi}=3 \theta$.

For relativistic phase velocities $\omega / c k \simeq 1$, the wavelength of the plasma oscillation given by Eq. (43) is

$$
\lambda=\lambda_{p} \gamma_{\perp}^{1 / 2}\left[1-\frac{\theta}{4 \gamma_{\perp}^{2}}-\frac{3 a^{2}}{4 \gamma_{\perp}^{2}} \theta\left(1-\gamma_{\perp}^{-2}\right)\right],
$$

where $\lambda_{p}=2 \pi c / \omega_{p}$. In the absence of a laser field $a=0$, $\lambda=\lambda_{p}(1-\theta / 4)$ and the wavelength of the oscillation is 
reduced from the plasma wavelength owing to the thermal inertia.

In the weakly-relativistic limit $a \ll 1$, neglecting terms of order $\theta a^{2} \ll 1$, yields the dispersion relation

$$
\omega^{2}=\omega_{p}^{2}\left(1-a^{2} / 2-5 \theta / 2\right)+3 k^{2} c^{2} \theta .
$$

Equation (46) is the relativistic Bohm-Gross dispersion relation including the lowest order correction to the relativistic plasma frequency owing to the quiver velocity.

In the absence of a laser field $(a=0)$, Eq. (43) reduces to the relativistic (including thermal inertia) Bohm-Gross dispersion relation derived by Clemmow and Wilson [27]: $\omega^{2}=\omega_{p}^{2}(1-5 \theta / 2)+3 \theta k^{2} c^{2}$.

\section{QUASI-STATIC WARM FLUID EQUATIONS}

In this section the warm fluid model is applied to describe the nonlinear response of the plasma to an intense laser field. It is assumed that the plasma wave is only a function of the co-moving variable $\xi=z-\beta_{\varphi} c t$, i.e., the quasi-static approximation (QSA) [2], where $\beta_{\varphi}$ is the phase velocity of the plasma wave (approximately the group velocity of the driver). In the QSA, the phasespace distribution takes the form $f\left(\xi, p^{\mu}\right)$. Here the laser driver is also assumed to be only a function of the comoving variable $a_{\perp}(\xi)$.

Applying the QSA, the continuity equation Eq. (8), $\partial_{\mu}\left(h u^{\mu}\right)=0$, becomes

$$
\partial_{\xi}\left[h \gamma_{\mathrm{th}} \Gamma\left(\beta_{\varphi}-w_{z}\right)\right]=0,
$$

or, for an initially quiescent plasma,

$$
h / n_{0}=\left[\gamma_{\mathrm{th}} \Gamma\left(1-\beta_{\varphi}^{-1} w_{z}\right)\right]^{-1} .
$$

The proper density is given by $n_{p}=\gamma_{\mathrm{th}} h$, and the density in the lab frame is $n=J^{0}=h u^{0}=\gamma_{\mathrm{th}} \Gamma h$. Hence, in the QSA, the plasma density in the lab frame is $n / n_{0}=$ $\left(1-\beta_{\varphi}^{-1} w\right)^{-1}$.

\section{A. Momentum variance evolution}

Applying the QSA, the momentum variance components [cf. Eqs. (34)-(36)] are $\Theta^{33} / n_{0}=\left(h / n_{0}\right) \theta_{33}$, $\Theta^{23} / n_{0}=\left(h / n_{0}\right) \theta_{23}, \Theta^{22} / n_{0}=\left(h / n_{0}\right) \theta_{22}$,

$$
\begin{aligned}
\frac{\Theta^{13}}{n_{0}} & =\Gamma\left(\frac{h}{n_{0}}\right)^{2}\left[\theta_{13}+\left(\frac{a_{\perp}}{\beta_{\varphi} \Gamma}\right) \theta_{23}\right], \\
\frac{\Theta^{12}}{n_{0}} & =\Gamma\left(\frac{h}{n_{0}}\right)^{2}\left[\theta_{12}+\left(\frac{a_{\perp}}{\beta_{\varphi} \Gamma}\right) \theta_{22}\right], \\
\frac{\Theta^{11}}{n_{0}} & =\Gamma^{2}\left(\frac{h}{n_{0}}\right)^{3}\left[\theta_{11}+2\left(\frac{a_{\perp}}{\beta_{\varphi} \Gamma}\right) \theta_{12}+\left(\frac{a_{\perp}}{\beta_{\varphi} \Gamma}\right)^{2} \theta_{22}\right],
\end{aligned}
$$

where $\theta_{i j}$ are constants of integration (the initial temperatures, normalized to $m c^{2} / k_{\mathrm{B}}$, assuming an initially quiescent plasma). Note that, for a large amplitude plasma wave, the peak of the density perturbation is nonlinear such that $h / n_{0} \gg 1$ and $\Theta_{33} \sim \Theta_{23} \sim \Theta_{22} \ll \Theta_{12} \sim$ $\Theta_{13} \ll \Theta_{11}$, i.e., the momentum variance tensor is highly anisotropic, and for highly nonlinear plasma waves with relativistic phase velocities, the longitudinal component of the momentum variance dominates. In the limit of $1 \mathrm{D}$ fluid motion, $\theta_{3 i}=\theta_{2 i}=0$ for $i=1,2,3$, and Eq. (51) reduces to Eq. (28).

For a plasma with an initially isotropic temperature, the constants of integration are $\theta_{i j}=\theta$ for $i=j$ and $\theta_{i j}=0$ for $i \neq j$. The variance tensor components for an initially isotropic plasma are $\Theta^{23}=\Theta^{13}=0$, and

$$
\begin{aligned}
\frac{\Theta^{22}}{n_{0}} & =\frac{\Theta^{33}}{n_{0}}=\frac{\left(1-w_{z}^{2}\right)^{1 / 2}}{\gamma_{\perp}\left(1-\beta_{\varphi}^{-1} w_{z}\right)} \theta, \\
\frac{\Theta^{12}}{n_{0}} & =\frac{\left(1-w_{z}^{2}\right)^{1 / 2}}{\gamma_{\perp}\left(1-\beta_{\varphi}^{-1} w_{z}\right)^{2}}\left[\frac{a_{\perp}}{\gamma_{\perp}} \beta_{\varphi}^{-1}\left(1-w_{z}^{2}\right)^{1 / 2}\right] \theta, \\
\frac{\Theta^{11}}{n_{0}} & =\frac{\left(1-w_{z}^{2}\right)^{1 / 2}}{\gamma_{\perp}\left(1-\beta_{\varphi}^{-1} w_{z}\right)^{3}}\left[1+\frac{a_{\perp}^{2}}{\gamma_{\perp}^{2}} \beta_{\varphi}^{-2}\left(1-w_{z}^{2}\right)\right] \theta,
\end{aligned}
$$

where $\gamma_{\perp}^{2}=1+a_{\perp}^{2}$. In the following, an isotropic initial temperature distribution is considered.

\section{B. Transverse momentum evolution}

For plasma fluid motion in a laser with fields given by Eqs. (31)-(33), the equations for the transverse momentum components, Eq. (25), reduce to

$$
\begin{gathered}
h \gamma_{\mathrm{th}} \Gamma u^{\mu} \partial_{\mu}\left(\gamma_{\mathrm{th}} \Gamma w_{\perp}-a_{\perp}\right)+\partial_{\mu} \Theta^{\mu 2}=0, \\
h \gamma_{\mathrm{th}} \Gamma u^{\mu} \partial_{\mu}\left(\gamma_{\mathrm{th}} \Gamma w_{3}\right)+\partial_{\mu} \Theta^{\mu 3}=0 .
\end{gathered}
$$

For an initially cold plasma, $u^{\mu} \partial_{\mu}\left(\Gamma w_{\perp}-a_{\perp}\right)=0$ and $u^{\mu} \partial_{\mu}\left(\Gamma w_{3}\right)=0$, i.e., canonical transverse fluid momentum is conserved. In the QSA, the transverse momentum equations Eqs. (55) and (56) become

$$
\partial_{\xi}\left[\gamma_{\mathrm{th}} \Gamma w_{\perp}-a_{\perp}-\beta_{\varphi}^{-1}\left(1-\beta_{\varphi} w_{z}\right) \frac{\Theta^{12}}{n_{0}}+w_{\perp} \frac{\Theta^{22}}{n_{0}}\right]=0,
$$

$$
\partial_{\xi}\left[\gamma_{\mathrm{th}} \Gamma w_{3}-\beta_{\varphi}^{-1}\left(1-\beta_{\varphi} w_{z}\right) \frac{\Theta^{13}}{n_{0}}+w_{\perp} \frac{\Theta^{23}}{n_{0}}\right]=0 .
$$

Assuming an initially isotropic, quiescent plasma, $\Theta^{13}=$ $\Theta^{23}=0, \partial_{\xi}\left(\gamma_{\mathrm{th}} \Gamma w_{3}\right)=0$, and Eq. (57) can be integrated to yield, to order $\mathcal{O}\left(\epsilon^{2}\right)$,

$$
\begin{gathered}
\Gamma w_{\perp}=a_{\perp}+\frac{\Theta^{12}}{n_{0}}\left[\Gamma w_{z} w_{\perp}^{2} \frac{n_{0}}{h}+\beta_{\varphi}^{-1}\left(1-\beta_{\varphi} w_{z}\right)\right] \\
-\frac{\Theta^{11}}{n_{0}}\left[\frac{1}{2} \Gamma w_{\perp} \frac{n_{0}}{h}\left(1-w_{z}^{2}\right)\right] \\
-\frac{\Theta^{22}}{n_{0}}\left[\frac{1}{2} \Gamma w_{\perp} \frac{n_{0}}{h}\left(1-w_{\perp}^{2}\right)+w_{\perp}\right]-\frac{\Theta^{33}}{n_{0}}\left[\frac{1}{2} \Gamma w_{\perp} \frac{n_{0}}{h}\right] .
\end{gathered}
$$


If $a_{\perp}=0$ (e.g., after the laser driver), then, to order $\mathcal{O}\left(\epsilon^{2}\right), \Gamma w_{\perp}=0$ and $\Gamma=\left(1-w_{z}^{2}\right)^{-1 / 2}$.

For the case of no momentum variance in the transverse direction $f=g\left(\xi, p_{z}\right) \delta^{2}\left(\boldsymbol{p}_{\perp}-\boldsymbol{a}_{\perp}\right)$, i.e., initially cold in the transverse direction such that $\theta_{33}=\theta_{23}=$ $\theta_{13}=\theta_{12}=\theta_{22}=0$, then

$$
\partial_{\xi}\left(\gamma_{\mathrm{th}} \Gamma w_{\perp}-a_{\perp}\right)=0
$$

which can be integrated to yield $\gamma_{\mathrm{th}} \Gamma w_{\perp}=a_{\perp}$, or

$$
w_{\perp}=\frac{a_{\perp}\left(1-w_{z}^{2}\right)^{1 / 2}}{\left(\gamma_{\mathrm{th}}^{2}+a_{\perp}^{2}\right)^{1 / 2}}=\frac{a_{\perp}\left(1-w_{z}^{2}\right)^{1 / 2}}{\left(\gamma_{\perp}^{2}+\epsilon^{2}\right)^{1 / 2}} .
$$

The case of a distribution with no transverse momentum variance, i.e., $f=g\left(\xi, p_{z}\right) \delta^{2}\left(\boldsymbol{p}_{\perp}-\boldsymbol{a}_{\perp}\right)$, was considered in Ref. [7].

\section{Energy-momentum evolution}

A quasi-static longitudinal constant of motion can be derived by considering the components of the energymomentum conservation equation Eq. (9),

$$
\begin{aligned}
& \partial_{\xi}\left(-\beta_{\varphi} T^{00}+T^{10}\right)=h \gamma_{\mathrm{th}} \Gamma\left(w_{z} \partial_{\xi} \phi+\beta_{\varphi} w_{\perp} \partial_{\xi} a_{\perp}\right) \\
& \partial_{\xi}\left(-\beta_{\varphi} T^{01}+T^{11}\right)=h \gamma_{\mathrm{th}} \Gamma\left(\partial_{\xi} \phi+w_{\perp} \partial_{\xi} a_{\perp}\right) .
\end{aligned}
$$

Combining Eqs. (62) and (63), and using the continuity equation Eq. (47), yields

$$
\begin{array}{r}
\partial_{\xi}\left(-\beta_{\varphi}^{2} T^{01}+\beta_{\varphi} T^{11}+\beta_{\varphi} T^{00}-T^{10}\right) \\
\quad=h \gamma_{\mathrm{th}} \Gamma\left(\beta_{\varphi}-w_{z}\right) \partial_{\xi} \phi=n_{0} \beta_{\varphi} \partial_{\xi} \phi
\end{array}
$$

Using $T^{\mu \nu}=h u^{\mu} u^{\nu}+\Theta^{\mu \nu}$, and Eq. (23) (i.e., $\Theta^{\mu 0}=$ $\left.w_{z} \Theta^{\mu 1}+w_{\perp} \Theta^{\mu 2}\right)$, Eq. (64) becomes

$$
\begin{gathered}
\partial_{\xi}\left[\left(h \gamma_{\mathrm{th}}^{2} \Gamma^{2}+\Theta^{11}\right)\left(1-\beta_{\varphi} w_{z}\right)\left(1-\beta_{\varphi}^{-1} w_{z}\right)+\Theta^{22} w_{\perp}^{2}\right. \\
\left.+\Theta^{12} w_{\perp}\left[2 w_{z}-\left(\beta_{\varphi}+\beta_{\varphi}^{-1}\right)\right]\right]=n_{0} \partial_{\xi} \phi
\end{gathered}
$$

Equation (65) yields the quasi-static longitudinal constant of motion for the distribution $f\left(\xi, p^{\mu}\right)$, assuming a plane laser field in an initially isotropic plasma. Assuming an initially quiescent plasma, Eq. (65) can be integrated to yield the longitudinal constant of motion:

$$
1+\phi+\frac{5}{2} \theta=\frac{\left(1-\beta_{\varphi} w_{z}\right) \gamma_{\perp}}{\left(1-w_{z}^{2}\right)^{1 / 2}}\left(1+\frac{\theta}{\gamma_{\perp}^{2}}\right)+\frac{3}{2} \theta\left(1+\frac{a_{\perp}^{2}}{\gamma_{\perp}^{2}} \beta_{\varphi}^{-2} \gamma_{\varphi}^{-2}\right) \frac{\left(1-\beta_{\varphi} w_{z}\right)\left(1-w_{z}^{2}\right)^{1 / 2}}{\gamma_{\perp}\left(1-\beta_{\varphi}^{-1} w_{z}\right)^{2}}
$$

with $\gamma_{\perp}^{2}=1+a_{\perp}^{2}$. The first term on the right-hand of Eq. (66) represents the kinetic energy of the plasma fluid motion in the wave $\left(\zeta=z-\beta_{\varphi} c t\right)$ frame. The terms proportional to $\theta$ represent the thermal contributions to the plasma kinetic energy. The left-hand side of Eq. (66) corresponds to the rest energy of the plasma, the electrostatic energy, and the energy contained in the initial thermal fluctuations. For $\theta=0$, Eq. (66) reduces to the well-known quasi-static result for the longitudinal constant of motion describing plasma wave excitation by an ultra-short laser pulse in a cold plasma [2].

Note that, in the QSA, the longitudinal momentum equation Eq. (25) can be written as

$$
\begin{aligned}
& \left(\beta_{\varphi}-w_{z}\right) \partial_{\xi} u_{z}=-\partial_{\xi} \phi-w_{\perp} \partial_{\xi} a_{\perp}+ \\
& \frac{n_{0}}{n} \partial_{\xi}\left[\left(1-\beta_{\varphi} w\right)\left(1+\frac{a_{\perp}^{2}}{\beta_{\varphi}^{2} \Gamma^{2}}\right) \frac{n^{3}}{n_{0}^{3} \Gamma}-a_{\perp}^{2} \frac{n^{2}}{n_{0}^{2} \Gamma^{3}}\right] \theta
\end{aligned}
$$

where $u_{z}=\gamma_{\mathrm{th}} \Gamma w_{z}, \Gamma=\left(1-w_{z}^{2}-w_{\perp}^{2}\right)^{-1 / 2}$, and $w_{\perp}$ is given by Eq. (59). The first term on the right-hand side of Eq. (67) is the electrostatic field of the plasma wave, the second term is responsible for the ponderomotive force of the laser pulse (with thermal corrections), and the third term is the thermal force.

\section{Temperature evolution}

In Appendix B it is shown that the invariant measure of thermal spread is equal to the width of a Maxwellian distribution (i.e., the temperature). The invariant measure of thermal spread $\epsilon^{2}=-\left(\Theta_{\mu}^{\mu} / h\right)$ evolves as

$$
\begin{aligned}
\epsilon^{2}=\frac{n_{0}}{h}\left[\left(1-w_{z}^{2}\right) \frac{\Theta^{11}}{n_{0}}+\right. & \left(1-w_{\perp}^{2}\right) \frac{\Theta^{22}}{n_{0}} \\
& \left.-2 w_{z} w_{\perp} \frac{\Theta^{12}}{n_{0}}+\frac{\Theta^{33}}{n_{0}}\right],
\end{aligned}
$$


and applying the QSA momentum variance equations yields

$$
\begin{array}{r}
\epsilon^{2}=\left[\frac{\left(1-w_{z}^{2}\right)}{\left(1-\beta_{\varphi}^{-1} w_{z}\right)^{2}}+2+\frac{2 a_{\perp}}{\gamma_{\perp}} \frac{\left(1-w_{z}^{2}\right)^{1 / 2}\left(1-\beta_{\varphi} w_{z}\right)}{\left(1-\beta_{\varphi}^{-1} w_{z}\right)^{2}}\right. \\
\left.\quad-\frac{a_{\perp}^{2}}{\gamma_{\perp}^{2}}\left(1-w_{z}^{2}\right)\left(1-\frac{\left(1-w_{z}^{2}\right)}{\left(\beta_{\varphi}-w_{z}\right)^{2}}\right)\right] \theta . \quad \text { (69) }
\end{array}
$$

Without the laser field (e.g., after the drive laser pulse, $a_{\perp}=0$ ), Eq. (69) simplifies to

$$
\epsilon^{2}=\left[\frac{\left(1-w_{z}^{2}\right)}{\left(1-\beta_{\varphi}^{-1} w_{z}\right)^{2}}\right] \theta+2 \theta .
$$

For nonlinear plasma waves, such that $h / n_{0} \gg 1$, the temperature is well-approximated by the $1 \mathrm{D}$ result $\epsilon^{2} \simeq$ $\left(h / n_{0}\right)^{2} \theta \simeq\left(n / \Gamma n_{0}\right)^{2} \theta$.

\section{NONLINEAR ELECTRON PLASMA WAVES}

The evolution of the plasma wave potential is determined by the Poisson equation

$$
k_{p}^{-2} \partial_{\xi}^{2} \phi=J^{0} / n_{0}-1=\gamma_{\mathrm{th}} \Gamma h / n_{0}-1 .
$$

The longitudinal constant of motion Eq. (66) can be combined with the continuity equation Eq. (48) and the Poisson equation Eq. (71) to yield the evolution equation for the plasma fluid momentum:

$$
\frac{\partial^{2}}{\partial k_{p}^{2} \xi^{2}} \phi\left(w_{z}\right)=\frac{w_{z}}{\beta_{\varphi}-w_{z}},
$$

with $\phi\left(w_{z}\right)$ given by Eq. (66). The excited plasma wave electric field is given by $\hat{E}=E_{z} / E_{0}=-k_{p}^{-1} \partial_{\xi} \phi\left(w_{z}\right)$, where $E_{0}=m c^{2} k_{p} / e$. In terms of the axial fluid velocity, the plasma density perturbation is $n / n_{0}-1=$ $w_{z} /\left(\beta_{\varphi}-w_{z}\right)$, and the plasma temperature evolution is given by Eq. (69). Equation (72) is an ordinary differential equation, which may be solved numerically for an arbitrary laser field $a_{\perp}(\xi)$. Equations (66) and (72) indicate that thermal effects will be a small contribution to the plasma response, of order $\theta \ll 1$, until the plasma density perturbation becomes sufficiently large.

Note that Eqs. (66) and (72) differ from the result of Ref. [18] (cf. Eqs. (4.7) and (4.10) of Ref. [18]). This is because, in this work, we have not a priori assumed isotropy of the momentum variance. Isotropy can only be assumed if the collisional frequency is much greater than the plasma frequency (i.e., the laser-plasma interaction time) [28]. For short-pulse (sub-ps) laser interaction in underdense plasma, the plasma wave excitation occurs on a time scale much shorter than the collisional time scale $(\sim \mathrm{ns})$, and homogeneity of the pressure tensor cannot be assumed. It should also be noted that the fluid equations derived in Ref. [18] do not reduce to the Bohm-Gross dispersion relation for a Langmuir wave upon linearization owing to the isotropic pressure assumption.

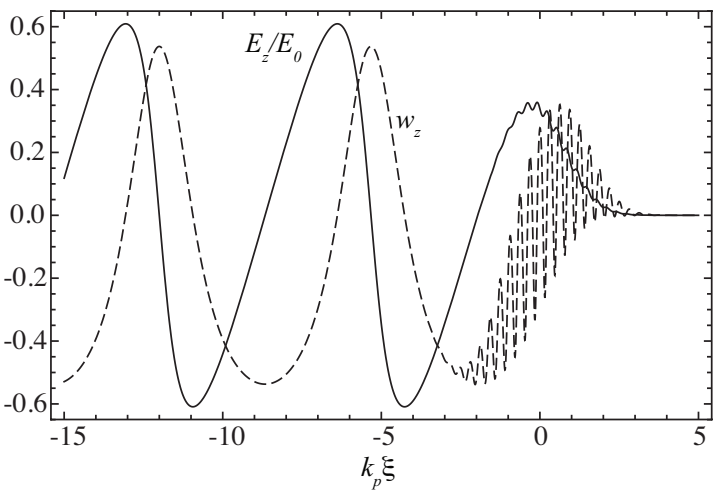

FIG. 2. Plasma wave axial fluid velocity $w_{z}$ (dashed curve) and electric field $E_{z} / E_{0}$ (solid curve) driven by a linearlypolarized Gaussian laser pulse with peak normalized intensity $a_{0}=1.5$ and intensity rms length $k_{p} L_{\mathrm{rms}}=1$ (centered at $k_{p} \xi=0$ moving toward the right) propagating in a plasma with $\omega_{0} / \omega_{p}=10$ and $\theta=10^{-4}$.

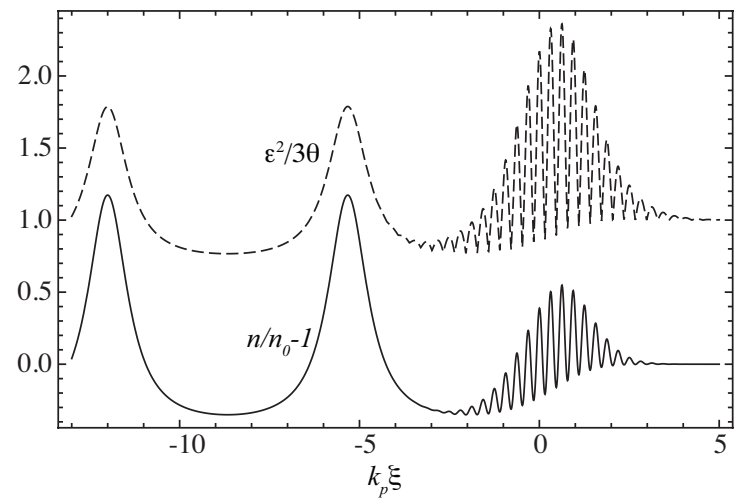

FIG. 3. Electron plasma density perturbation $n / n_{0}-1$ (solid curve) and normalized plasma temperature $\epsilon^{2} /(3 \theta)$ (dashed curve) for the same laser-plasma parameters as Fig. 2.

Figure 2 shows the plasma wave fluid velocity $w_{z}$ (dashed curve) and the plasma wave electric field $E_{z} / E_{0}$ (solid curve) excited by a linearly-polarized Gaussian laser pulse with envelope $a_{\perp}=a_{0} \exp \left(-\xi^{2} / 4 L_{\mathrm{rms}}^{2}\right), a_{0}=$ 1.5 , and intensity rms length $k_{p} L_{\mathrm{rms}}=1$, in a plasma with $\omega_{0} / \omega_{p}=10$ and $\theta=10^{-4}$. Figure 2 illustrates the relativistic motion $w_{z} \sim 1$ and the nonlinear steepening of the plasma wave electric field. Figure 3 shows the electron plasma density perturbation $\left(n / n_{0}-1\right)$ and the evolution of the normalized plasma temperature $\epsilon^{2} /(3 \theta)$. The plasma temperature undergoes periodic oscillations in the wake owing to compression of the plasma electron density. Figure 4 shows the anisotropic momentumdensity variance tensor components $\Theta^{\mu \nu}$ in the presence of an intense laser field (owing to coupling between the longitudinal and transverse momentum spread via the transverse laser field). This example corresponds to typical parameters for laser-plasma accelerator experiments using a short-pulse, intense Ti:Sapphire laser in a gas jet, e.g., a $0.8 \mu \mathrm{m}$ laser of peak intensity $4.8 \times 10^{18} \mathrm{~W} / \mathrm{cm}^{2}$ 


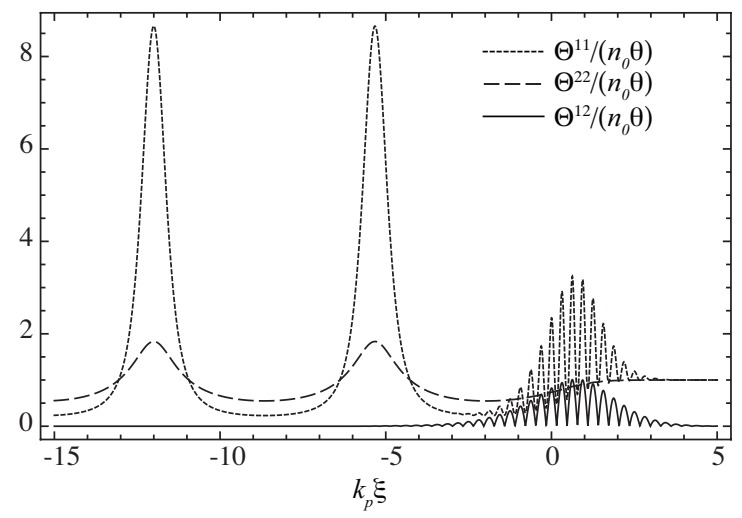

FIG. 4. Momentum-density variance components (normalized to $\left.n_{0} \theta\right) \Theta^{11}$ (dotted curve), $\Theta^{22}=\Theta^{33}$ (dashed curve), and $\Theta^{12}$ (solid curve) for the same laser-plasma parameters as Fig. 2.

and FWHM intensity duration of 10 fs propagating in a uniform photo-ionized (temperature $\sim 10 \mathrm{eV}$ ) plasma with electron number density $1.7 \times 10^{19} \mathrm{~cm}^{-3}$. For this density, $E_{0}=m c \omega_{p} / e \simeq 4 \times 10^{11} \mathrm{~V} / \mathrm{m}$. The plasma density perturbation can be measured experimentally via interferometric techniques, and frequency domain holography [29] has been successfully applied to measure plasma waves with relativistic phase velocities.

Linearizing Eq. (72) $\left(w_{z} \ll 1\right.$ and assuming $a_{\perp} \simeq$ constant) and taking the Fourier transform $\partial_{\xi} \rightarrow i k$ yields the generalized dispersion relation Eq. (43), and the relativistic Bohm-Gross dispersion relation in the absence of a laser field $\left(a_{\perp}=0\right)$.

Assuming a plasma wave with relativistic phase velocity $\left(\beta_{\varphi} \simeq 1\right)$ and linearizing Eq. (72) in the weaklyrelativistic regime $\left(a_{\perp} \ll 1\right)$ yields the wave equation driven by the ponderomotive force

$$
\left[\partial_{\xi}^{2}+k_{p}^{2}(1+\theta / 2)\right] w_{z}=\partial_{\xi}^{2} a_{\perp}^{2} / 2 .
$$

In the linear, weakly-relativistic regime $a_{\perp}^{2} \ll 1$, the dominant thermal effect is a change in the wavelength of the plasma wave $\lambda \simeq \lambda_{p}(1-\theta / 4)$.

\section{A. First integral of plasma wave equation}

Consider a plasma wave after a short laser driver (e.g., the standard laser wakefield regime) where $\gamma_{\perp}=1$, or a plasma wave excited by a long laser pulse (e.g., the self-modulated laser wakefield regime) such that $\gamma_{\perp}^{-1}\left|k_{p}^{-1} \partial_{\xi} \gamma_{\perp}\right| \ll 1$ and $\gamma_{\perp} \simeq$ constant. For these cases, the first integral of Eq. (72) may be evaluated. It is convenient to introduce the variables

$$
\tau=(3 / 2) \gamma_{\perp}^{-2}\left(1+a_{\perp}^{2} \gamma_{\perp}^{-2} \beta_{\varphi}^{-2} \gamma_{\varphi}^{-2}\right) \theta
$$

with $\tau \ll 1$, and $\chi^{2}=\left(1-w_{z}\right) /\left(1+w_{z}\right)$ such that $w_{z}=\left(1-\chi^{2}\right) /\left(1+\chi^{2}\right),\left(1-w_{z}^{2}\right)^{-1 / 2}=\left(1+\chi^{2}\right) /(2 \chi)$, and the longitudinal constant of motion Eq. (66) may be rewritten as

$$
\begin{aligned}
1+\phi=\frac{\gamma_{\perp}}{2 \chi} & {\left[\left(1-\beta_{\varphi}\right)+\left(1+\beta_{\varphi}\right) \chi^{2}\right] } \\
\times & \left\{1+\frac{4 \beta_{\varphi}^{2} \tau \chi^{2}}{\left[\left(1-\beta_{\varphi}\right)-\left(1+\beta_{\varphi}\right) \chi^{2}\right]^{2}}\right\},
\end{aligned}
$$

where $\theta \ll 1$ is assumed.

The plasma wave electric field evolution can be written as $k_{p}^{-1} \partial_{\xi}\left(\hat{E}^{2}\right)=2\left(k_{p}^{-1} \partial_{\xi} \hat{E}\right) \hat{E}=2\left[w_{z} /\left(\beta_{\varphi}-\right.\right.$ $\left.\left.w_{z}\right)\right]\left(k_{p}^{-1} \partial_{\xi} \phi\right)$, or

$$
\frac{d \hat{E}^{2}}{d \chi}=\left[\frac{2\left(1-\chi^{2}\right)}{\beta_{\varphi}\left(1+\chi^{2}\right)-\left(1-\chi^{2}\right)}\right] \frac{d \phi}{d \chi}
$$

Using Eq. (75), the above equation can be integrated to yield the electric field as a function of the fluid velocity:

$$
\hat{E}^{2}=\gamma_{\perp}\left(\chi_{i}-\chi+\chi_{i}^{-1}-\chi^{-1}+\left[\tilde{F}\left(\chi_{i}\right)-\tilde{F}(\chi)\right] \tau\right),
$$

where

$$
\tilde{F}(\chi)=\frac{4 \beta_{\varphi}^{2} \chi\left[\left(1-\chi^{4}\right)-\beta_{\varphi}\left(\chi^{4}-2 \chi^{2} / 3+1\right)\right]}{\left[\left(1-\beta_{\varphi}\right)-\left(1+\beta_{\varphi}\right) \chi^{2}\right]^{3}},
$$

and $\chi_{i}$ is given by the initial condition (determined by the laser driver). Equation (77) is a general expression for the electric field as a function of $\chi=\left(1-w_{z}\right) /\left(1+w_{z}\right)$ given by the initial excitation $\chi_{i}$.

\section{B. Maximum plasma wave electric field}

The peak of the electric field oscillation occurs at the phase such that $\partial_{\xi} \hat{E}=0$. Using the Poisson equation,

$$
k_{p}^{-1} \partial_{\xi} \hat{E}=\frac{-\left(1-\chi^{2}\right)}{\beta_{\varphi}\left(1+\chi^{2}\right)-\left(1-\chi^{2}\right)},
$$

and the phase location of the peak of the field oscillation $\partial_{\xi} \hat{E}=0$ occurs at the momentum $\chi=1$ (i.e., $w_{z}=$ $0)$. From the first integral of the plasma wave equation Eq. (77), the peak field, given the initial condition $\chi_{i}$, is $\hat{E}_{\text {peak }}=\hat{E}\left(\chi_{i}, \chi=1\right)$.

The maximum plasma wave electric field possible (sometimes referred to as the "wavebreaking" field), is given by $\hat{E}\left(\chi_{0}, \chi=1\right)$, where $\chi_{0}$ is the initial condition such that $d \hat{E}\left(\chi_{i}, \chi=1\right) / d \chi_{i}=0$. Using Eqs. (77) and (76), the maximum initial momentum is given by the momentum $\chi_{0}$ that produces the extremum of $\phi$. Using Eq. (75) and solving $d \phi /\left.d \chi\right|_{\chi=\chi_{0}}=0$ (i.e., a quartic equation for $\chi_{0}^{2}$ ), yields the momentum which produces 


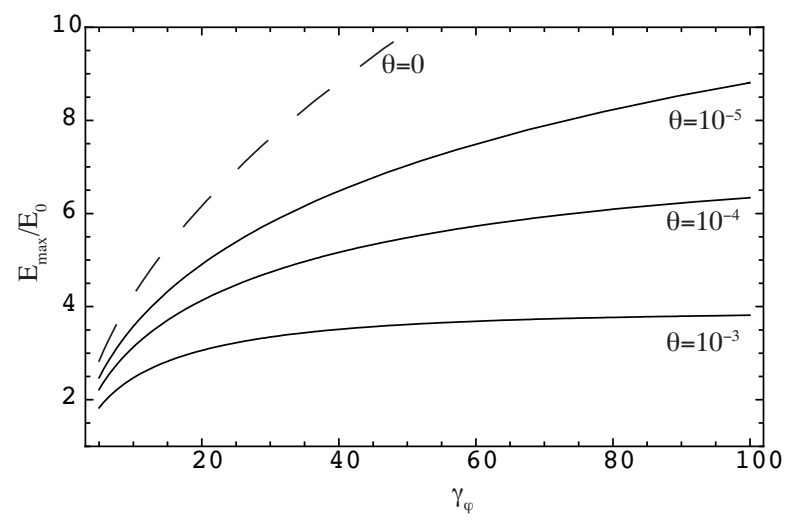

FIG. 5. Maximum electric field amplitude $\hat{E}_{\max }=E_{\max } / E_{0}$ [Eq. (81)] versus $\gamma_{\varphi}$ with $\gamma_{\perp}=1$. Dashed line is the cold limit $\theta=0$.

the extremum,

$$
\begin{aligned}
\chi_{0}^{2} & =\left(1+\beta_{\varphi}\right)^{-2}\left\{\gamma_{\varphi}^{-2}+\beta_{\varphi}^{2} \tau\right. \\
& +\beta_{\varphi} \sqrt{\tau}\left(8 \gamma_{\varphi}^{-2}+\beta_{\varphi}^{2} \tau\right)^{1 / 2}+\left[2 \tau \beta_{\varphi}^{2}\left(5 \gamma_{\varphi}^{-2}+\beta_{\varphi}^{2} \tau\right)\right. \\
& \left.\left.+2 \beta_{\varphi}\left(\gamma_{\varphi}^{-2}+\beta_{\varphi}^{2} \tau\right)\left(8 \gamma_{\varphi}^{-2} \tau+\beta_{\varphi}^{2} \tau^{2}\right)^{1 / 2}\right]^{1 / 2}\right\} .
\end{aligned}
$$

where $\gamma_{\varphi}=\left(1-\beta_{\varphi}^{2}\right)^{-1 / 2}$. In the cold limit $\tau=0, \chi_{0}^{2}=$ $\left(1-\beta_{\varphi}\right) /\left(1+\beta_{\varphi}\right)$ (i.e., $\left.w_{z}=\beta_{\varphi}\right)$, and the extremum of the potential occurs when the fluid velocity equals the phase velocity of the wave. Note that, for $\beta_{\varphi} \leq 1, \chi_{0}\left(\beta_{\varphi}\right) \geq$ $\tau^{1 / 2}$, and this indicates that the plasma wave amplitude is always bounded (including the case with phase velocity approaching the speed of light).

Evaluating Eq. (77) at the phase such that $\chi=1$ and the initial excitation such that $\chi_{i}=\chi_{0}$ yields the maximum plasma wave electric field amplitude

$$
\hat{E}_{\max }^{2}=\gamma_{\perp}\left(\chi_{0}+\chi_{0}^{-1}-2+\left[\tilde{F}\left(\chi_{0}\right)-2 / 3\right] \tau\right),
$$

where $\tilde{F}\left(\chi_{0}\right)$ is given by Eqs. (78) and (80). Physically, this limit on the plasma wave amplitude is due to the pressure force. As the plasma becomes highly compressed, the pressure force grows, ultimately limiting the density compression and therefore the wave amplitude. For sufficiently large drive intensity, no traveling wave solutions exist. Figure 5 shows the maximum field amplitude of the plasma wave as a function of phase velocity $\left(\gamma_{\varphi}\right)$, and Fig. 6 shows the maximum field amplitude as a function of initial plasma temperature $\theta$. Also shown is the cold result (dashed curve in Fig. 5) and the ultrahigh phase velocity result (dashed curve in Fig. 6). Thermal effects reduce the maximum amplitude from the cold wavebreaking result.

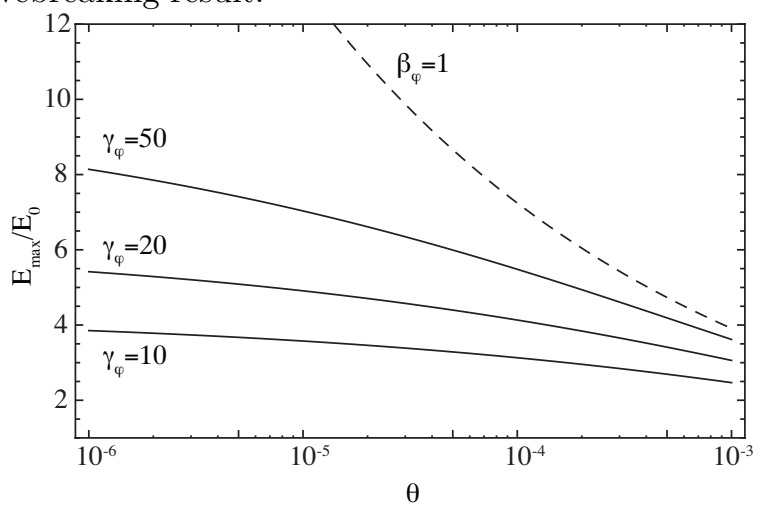

FIG. 6. Maximum electric field amplitude $\hat{E}_{\max }=E_{\max } / E_{0}$ [Eq. (81)] versus initial temperature $\theta$ with $\gamma_{\perp}=1$. Dashed line is the limit $\beta_{\varphi}=1$.

\section{Cold plasma limit}

In the cold plasma limit $\tau=0, \chi_{0}=\gamma_{\varphi}\left(1-\beta_{\varphi}\right)$, and the maximum electric field is

$$
\hat{E}_{\max }^{2}=\gamma_{\perp}\left(\chi_{0}+\chi_{0}^{-1}-2\right)=2 \gamma_{\perp}\left(\gamma_{\varphi}-1\right) .
$$

Equation (82) is a generalization of the cold relativistic wavebreaking field to include the presence of a laser field [7]. Equation (82) indicates that the maximum field amplitude inside a laser field can be significantly larger compared to after the drive laser pulse (where $a_{\perp}=0$ and $\left.\gamma_{\perp}=1\right)$.

\section{Maximum field amplitude of laser-driven plasma waves}

A plasma wave driven by a short-pulse laser will have a phase velocity approximately equal to the group velocity of the laser pulse, typically $\gamma_{\varphi} \sim 10-100$ for laser propagation in an underdense plasma. Without some additional heating mechanism, laboratory plasmas used for laser-plasma accelerator experiments have temperatures of the order of the ionization potential, $m c^{2} \theta \sim 10 \mathrm{eV}$ $[11,12]$. Therefore laser-driven plasma waves typically satisfy $\theta \gamma_{\varphi}^{2} \ll 1$.

In the limit, $\theta \gamma_{\varphi}^{2} \ll \beta_{\varphi}^{2}$, Eq. (81) may be expanded to yield

$$
\hat{E}_{\max }^{2} \simeq 2 \gamma_{\perp}\left(\gamma_{\varphi}-1\right)-\gamma_{\varphi} \gamma_{\perp} \beta_{\varphi}^{2}\left[\frac{8}{3}\left(\frac{2 \tau \gamma_{\varphi}^{2}}{\beta_{\varphi}^{2}}\right)^{1 / 4}-2\left(\frac{2 \tau \gamma_{\varphi}^{2}}{\beta_{\varphi}^{2}}\right)^{1 / 2}+\beta_{\varphi}^{2}\left(\frac{2 \tau \gamma_{\varphi}^{2}}{\beta_{\varphi}^{2}}\right)^{3 / 4}+\left(\frac{1}{3 \gamma_{\varphi}^{3}}-\frac{\beta_{\varphi}^{2}}{4}\right)\left(\frac{2 \tau \gamma_{\varphi}^{2}}{\beta_{\varphi}^{2}}\right)\right]
$$


For the case $\gamma_{\varphi} \gg 1$, Eq. (83) reduces to

$$
\frac{\hat{E}_{\max }^{2}}{\gamma_{\perp} \gamma_{\varphi}} \simeq 2\left(1-\gamma_{\varphi}^{-1}\right)-\frac{8}{3}\left(2 \gamma_{\varphi}^{2} \tau\right)^{1 / 4}+2\left(2 \gamma_{\varphi}^{2} \tau\right)^{1 / 2},
$$

to lowest order assuming $\gamma_{\varphi}^{2} \tau \ll 1$ (and $\gamma_{\varphi}^{6} \tau \gg 1$ ). Equation (84) contains the cold relativistic wavebreaking field (generalized to include the influence of the laser) with the lowest order corrections owing to the plasma temperature. For relativistic phase velocities $\gamma_{\varphi} \gg 1$ driven by intense laser pulses $\left(a_{\perp} \sim 1\right), \tau \simeq(3 / 2) \theta / \gamma_{\perp}^{2}$ and Eq. (84) reduces to the result of Ref. [7].

For plasma waves with relativistic phase velocities $\gamma_{\varphi} \gg 1$, the warm fluid theory predicts the maximum longitudinal velocity $w_{z, \max } \simeq \beta_{\varphi}\left[1-\gamma_{\varphi}^{-3 / 2}(2 \tau)^{1 / 4}-\right.$ $\left.\gamma_{\varphi}^{-1}(2 \tau)^{1 / 2}\right]<\beta_{\varphi}$; the fluid velocity never reaches the phase velocity of the plasma wave. And, at the maximum field amplitude $\hat{E}=\hat{E}_{\max }$, the maximum longitudinal fluid momentum is $u_{z, \max } \simeq \gamma_{\varphi}\left[1-\left(2 \tau \gamma_{\varphi}^{2}\right)^{1 / 4}\right] \simeq$ $\gamma_{\varphi}-3^{1 / 2}\left(\Theta^{11} / h\right)_{\max }^{1 / 2}$, for $\gamma_{\varphi} \gg 1$ and $\gamma_{\varphi}^{2} \tau \ll 1$, i.e., the difference between the maximum fluid momentum and $\gamma_{\varphi}$ is of the order of the momentum variance. The above results imply a relation between the maximum plasma wave amplitude ("wavebreaking") and particle trapping in the plasma wave. Consider the regime of laser-driven plasma waves $\left(\gamma_{\varphi}^{2} \tau \ll 1\right.$ and $\left.\gamma_{\varphi}^{2} \gg 1\right)$ in the region behind the laser driver $\left(a_{\perp}^{2}=0\right)$, at $\hat{E}=\hat{E}_{\max }$, the peak value of the longitudinal fluid velocity is $w_{\max }=$ $\beta_{\varphi}-\gamma_{\varphi}^{-3 / 2}(3 \theta)^{1 / 4}$ and the peak value of the longitudinal fluid momentum is $u_{z, \max }=\gamma_{\varphi}-\gamma_{\varphi}^{3 / 2}(3 \theta)^{1 / 4}$. At the phase position where the longitudinal fluid velocity and momentum are at their peak, the plasma density is maximum. The longitudinal thermal velocity and momentum spread (variance) are $\beta_{\text {th }} \simeq \Gamma^{-3}\left(\Theta^{11} / h\right)^{1 / 2} \simeq \epsilon / \Gamma^{2}$ and $\left(\Theta^{11} / h\right)^{1 / 2} \simeq \Gamma \epsilon$, respectively, and at the phase position where the longitudinal fluid velocity is at its peak [cf. Eq. (91)] $\epsilon_{\max } \simeq \gamma_{\varphi}^{1 / 2}(\theta / 3)^{1 / 4}$. Hence, at the maximum amplitude, $\beta_{\varphi}-w_{\max } \simeq \sqrt{3} \beta_{\mathrm{th}}$. As the amplitude of the wave approaches $\hat{E}_{\text {max }}$, electrons in the tail of a typical plasma distribution may be trapped [30, 31]. Although the peak value of the mean fluid velocity is less than the phase velocity, $w_{\max }<\beta_{\varphi}$ (and $u_{z, \max }<\gamma_{\varphi} \beta_{\varphi}$ ), electrons on the tail of a thermal distribution with velocities in excess of the phase velocity will be trapped. Specifically, thermal electrons with velocities in excess of $\sqrt{3} \beta_{\text {th }}$. Assuming an initially Gaussian thermal distribution, this corresponds to approximately $4 \%$ of the electron population are continuously being trapped by the plasma wave with amplitude $\hat{E}_{\max }$ [31]. Once a significant number of electrons become trapped in the plasma wave, the plasma wave amplitude becomes time dependent (no longer a function of only $z-\beta_{\varphi} c t$ ), owing to beam loading and damping of the plasma wave, and a purely traveling wave solution is no longer possible.

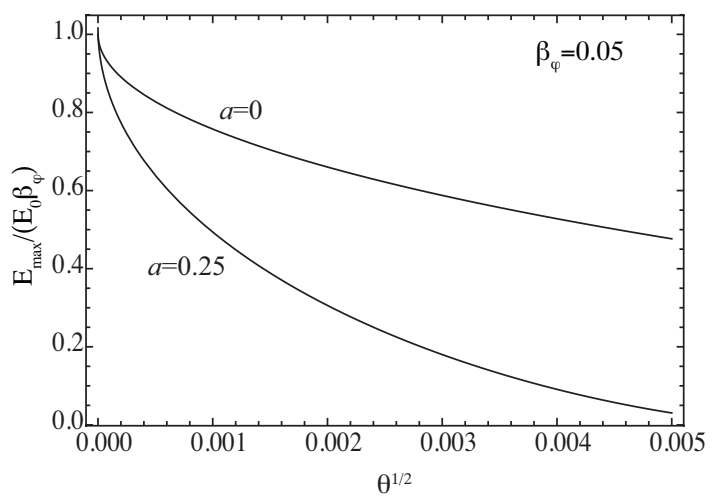

FIG. 7. Normalized maximum plasma wave amplitude $\beta_{\varphi}^{-1} \hat{E}_{\max }$ as function of initial thermal velocity $\theta^{1 / 2}$ for $a_{\perp}=0$ and $a_{\perp}=0.25$ with non-relativistic phase velocity $\beta_{\varphi}=0.05$

\section{Nonrelativistic phase velocities}

For a non-relativistic plasma wave phase velocity, such that $\tau \ll \beta_{\varphi}^{2} \ll 1$, Eq. (83) reduces to

$$
\frac{\hat{E}_{\max }^{2}}{\gamma_{\perp} \beta_{\varphi}^{2}} \simeq 1-\frac{8}{3}\left(\frac{2 \tau}{\beta_{\varphi}^{2}}\right)^{1 / 4}+2\left(\frac{2 \tau}{\beta_{\varphi}^{2}}\right)^{1 / 2}-\frac{1}{3}\left(\frac{2 \tau}{\beta_{\varphi}^{2}}\right)
$$

with $\tau=(3 / 2)\left(\theta / \gamma_{\perp}^{2}\right)\left(1+a_{\perp}^{2} \beta_{\varphi}^{-2} \gamma_{\perp}^{-2}\right)$, where terms of the order $\mathcal{O}\left(\tau \beta_{\varphi}^{2}\right)$ have been neglected. Equation (85) with $\gamma_{\perp}=1$ is the result derived by Coffey [10], which assumed a non-relativistic plasma wave and a waterbag momentum distribution. In the limit $a_{\perp}^{2} / \gamma_{\perp}^{2} \gg \beta_{\varphi}^{2}$ (with $\left.\theta \ll \beta_{\varphi}^{4} \ll 1\right)$,

$$
\frac{\hat{E}_{\max }^{2}}{\gamma_{\perp} \beta_{\varphi}^{2}} \simeq 1-\frac{8}{3}\left(\frac{3 \theta a_{\perp}^{2}}{\beta_{\varphi}^{4} \gamma_{\perp}^{4}}\right)^{1 / 4}+2\left(\frac{3 \theta a_{\perp}^{2}}{\beta_{\varphi}^{4} \gamma_{\perp}^{4}}\right)^{1 / 2}-\frac{1}{3}\left(\frac{3 \theta a_{\perp}^{2}}{\beta_{\varphi}^{4} \gamma_{\perp}^{4}}\right)
$$

As shown in Figs. 7 and 8 the presence of an intense laser field strongly modifies the maximum field amplitude for plasma waves with non-relativistic phase velocities. Figure 7 shows the maximum plasma wave amplitude $\beta_{\varphi}^{-1} \hat{E}_{\max }$ versus initial thermal velocity for nonrelativistic phase velocity $\beta_{\varphi}=0.05$. Shown in Fig. 7 is the reduction of the maximum plasma wave amplitude in the presence of an intense laser field with $a_{\perp}=0.25$ compared to the result of Ref. [10] (i.e., for $a_{\perp}=0$ ). This is due to the increased thermal spread via the transverse coupling of the momentum variance tensor. Figure 8 shows the maximum plasma wave amplitude $\beta_{\varphi}^{-1} \hat{E}_{\max }$ as function of laser field intensity for non-relativistic phase velocity $\beta_{\varphi}=0.1$ and initial temperature $\theta=10^{-5}$ as a function of laser intensity. For sufficiently large laser intensity $a_{\perp} \gtrsim 1$, the relativistic electron mass shift results in a larger maximum plasma wave amplitude. 


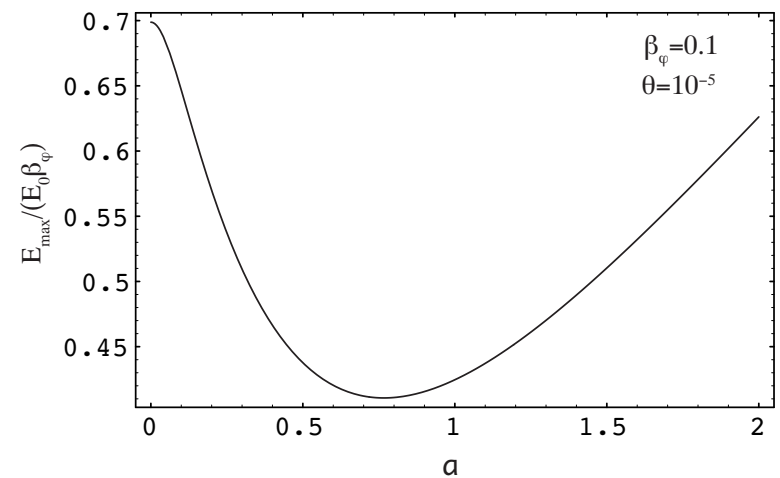

FIG. 8. Normalized maximum plasma wave amplitude $\beta_{\varphi}^{-1} \hat{E}_{\max }$ as function of laser field intensity $a_{\perp}$ for nonrelativistic phase velocity $\beta_{\varphi}=0.1$ and initial temperature $\theta=10^{-5}$.

\section{Nonlinear plasma wavelength}

The wavelength on the nonlinear plasma oscillation can be evaluated from the wave equation Eq. (72). The first integral of the plasma wave equation Eq. (77) can be expressed in the form $\hat{E}^{2} / 2+V(\phi)=V_{0}$, where the pseudopotential is

$$
V(\phi)=V(\chi(\phi))=\frac{1}{2}\left[\chi+\chi^{-1}+\tau \tilde{F}(\chi)\right],
$$

and $V_{0} \leq V\left(\chi_{i}\right)$ (the constant $V_{0}=V\left(\chi_{0}\right)$ corresponds to the maximum wave amplitude). The period of the plasma oscillation $\lambda_{\text {osc }}$ can be computed from $k_{p}^{-1} \partial_{\xi} \phi=$ $-\hat{E}=-\sqrt{2\left(V_{0}-V\right)}$, namely

$$
\lambda_{\mathrm{osc}}=\int d \xi=\frac{\lambda_{p}}{\pi} \int_{\chi_{\min }}^{\chi_{\max }} d \chi \frac{(d \phi / d \chi)}{\sqrt{2\left(V_{0}-V\right)}}
$$

where $\chi_{\min / \max }$ is given by the solutions to $V_{0}=V(\chi)$. At the maximum plasma wave amplitude, and assuming $\hat{E}_{\max }^{2} \gg 1, \lambda_{\text {osc }} / \lambda_{p} \simeq(2 / \pi) \hat{E}_{\max }$, where $\hat{E}_{\max }$ is given by Eq. (81). Thermal effects reduce the wavelength of the plasma oscillation.

\section{Plasma temperature at the maximum field amplitude}

The highly-compressed electron plasma density results in local increase in the plasma temperature (as shown in Fig. 3). From the continuity equation Eq. (47), the lab-frame plasma density is

$$
\frac{n}{n_{0}}=\frac{\beta_{\varphi}}{\beta_{\varphi}-w_{z}}=\frac{\beta_{\varphi}\left(1+\chi^{2}\right)}{\beta_{\varphi}\left(1+\chi^{2}\right)-\left(1-\chi^{2}\right)} .
$$

The peak density perturbation at the maximum field amplitude is given by $n\left(\chi_{0}\right) / n_{0}$, which does not become singular in contrast to the cold fluid theories (i.e., there is no shock formation).
Using Eq. (70), the invariant measure of thermal spread (i.e., the plasma temperature) is, after the laser pulse,

$$
\epsilon^{2}=\theta\left[\frac{2 \beta_{\varphi} \chi}{\beta_{\varphi}\left(1+\chi^{2}\right)-\left(1-\chi^{2}\right)}\right]^{2}+2 \theta
$$

and the maximum temperature occurs at the maximum compression, i.e., at $\chi=\chi_{0}$.

In the limit $\tau<\gamma_{\varphi}^{-2} \ll 1$ (e.g., laser-driven plasma wave), the temperature at the maximum field is

$$
\epsilon_{\max }^{2} \simeq \frac{1}{3} \gamma_{\perp}^{2}\left(2 \gamma_{\varphi}^{2} \tau\right)^{1 / 2}\left[1-\frac{1}{4}\left(2 \gamma_{\varphi}^{2} \tau\right)^{1 / 2}\right]
$$

and the temperature of the plasma remains nonrelativistic $\epsilon_{\max }^{2} \ll 1$. For a laser-driven plasma wave, the temperature of the plasma will remain non-relativistic at the maximum possible compression. This shows that the asymptotic expansion $\epsilon^{2}<1$ performed by the warm plasma assumption presented here is always valid, provided the initial temperature is non-relativistic (such that $\theta \gamma_{\varphi}^{2}<1$ in the laser-driven regime). The maximum density perturbation is finite and peaks at $\left(n / n_{0}\right)_{\max } \simeq$ $\gamma_{\varphi}\left(\beta_{\varphi} \gamma_{\varphi}\right)^{1 / 2}(2 \tau)^{-1 / 4} \gg 1$. In the limit of non-relativistic phase velocity $\beta_{\varphi} \ll 1$, the maximum temperature is $\epsilon_{\max }^{2} \simeq \beta_{\varphi}(2 \tau)^{1 / 2} / 3 \ll 1$.

In the limit, $\gamma_{\varphi}^{-2} \ll \theta \ll 1$ (e.g., a highly-relativistic particle beam-driven plasma wave), then $\epsilon_{\max }^{2}\left(\beta_{\varphi}=1\right)=$ $2 / 3$ and $\hat{E}_{\max }^{2}=(2 / 3)^{3 / 2} \theta^{-1 / 2}$. Note that, in this ultrarelativistic limit, the maximum field is independent of the phase velocity. This is in agreement with numerical solutions of the Vlasov equation in the limit $\gamma_{\varphi}^{2} \theta \gg 1$ that found $E_{\max }$ independent of $\gamma_{\varphi}$ [32]. In this limit, higher order moments of the distribution may contribute to the plasma response. This implies that the plasma response will be a function of the specific details of the form of the phase space distribution (i.e., the higher-order moments). An analysis of the maximum amplitude assuming a 3D water-bag momentum distribution has recently been carried out in Ref. [33], and it was shown that, in the limit $\beta_{\varphi} \rightarrow 1, \hat{E}_{\max }^{2}=(9 / 20)^{1 / 2} \theta^{-1 / 2}$. Note that the differences in the coefficient can be attributed to choice of a specific distribution in the case of the 3D water-bag assumption, or to the asymptotic expansion in the case of the warm fluid assumption.

\section{CONCLUSIONS AND DISCUSSION}

In this work, a relativistic warm fluid theory of a nonequilibrium, collisionless plasma was applied to model intense, short-pulse laser-plasma interactions. The fluid equations were treated asymptotically, and closure was obtained by assuming a non-relativistic temperature $\epsilon^{2} \ll 1$ (no specific form of the phase-space distribution $f$ or equation of state was assumed). The relativistic warm fluid theory was used derive the evolution of the 
momentum variance in the presence of an intense laser field (variance components given in Sec. III B). The momentum variance was found to be anisotropic and the presence of the laser field allowed coupling between the transverse and longitudinal components. A generalized Langmuir dispersion relation in the presence of an intense laser field Eq. (43) was derived from the linearized warm fluid equations.

A quasi-static wave equation Eq. (72) was derived [with $\phi\left(w_{z}\right)$ given by the constant of motion Eq. (66)], assuming $f\left(\xi, p_{\mu}\right)$, and used to describe nonlinear electron plasma waves excited by intense, short-pulse lasers. The quasi-static wave equation was used to calculate the maximum plasma wave amplitude assuming a travelling wave $\hat{E}_{\max }$. Thermal effects decreased the maximum wave amplitude compared to the cold result. For the case of relativistic phase velocities, the plasma wave evolution is dominated by the longitudinal momentum variance (and the maximum field amplitude reduces to the limit of 1D motion described in Ref. [7]). For the case of non-relativistic phase velocity plasma waves, the presence of a laser field can strongly modify the maximum field amplitude, owing to the coupling between transverse and longitudinal thermal fluctuations. The warm plasma theory remains finite at the maximum field amplitude, and does not contain the singular behavior observed assuming a cold plasma.

If the plasma temperature is relativistic, the asymptotic expansion described in Sec. III will no longer be valid. For relativistic temperatures, the higher-order moments of the distribution will be important and will be a function of the specific form of the phase-space distribution. Note that, the choice of an unphysical distribution (e.g., 1D water-bag) may lead to unphysical, singular (unbounded) solutions in the relativistic temperature regime, since details of the shape of the phase-space distribution (i.e., the higher-order moments) become important. For sufficiently large density perturbations, the collisionless plasma model will no longer be valid and collisional effects will become important.

In addition to the warm plasm assumption, waveparticle interactions were neglected in this theory. For example, particle trapping of a significant fraction of the distribution in a plasma wave can lead to broadening of the momentum spread such that the assumption $\epsilon^{2} \ll 1$ is no longer valid. The warm plasma assumption will be valid provided that the bulk of the plasma distribution is far from resonance with the wave.

As the field amplitude approaches and exceeds the maximum amplitude of a traveling wave (wavebreaking limit), particles in the tail of the plasma distribution may becomes trapped in the plasma wave [30, 31]. Specifically, for laser-driven plasma waves $\left(\gamma_{\varphi}^{2} \tau \ll 1\right.$ and $\gamma_{\varphi}^{2} \gg 1$ ), the results of the warm fluid theory imply that the maximum longitudinal fluid velocity $w_{\max }$ satisfies $w_{\max }+\sqrt{3} \beta_{\mathrm{th}} \simeq \beta_{\varphi}$, where $\beta_{\mathrm{th}}$ is the thermal velocity spread (variance) of the electron distribution. At the maximum field amplitude of a travelling wave, electrons in the tail of the distribution with velocities $>\sqrt{3} \beta_{\text {th }}$ will be trapped. When a sufficient number of electrons become trapped, the wave will be perturbed such that the quasi-static assumption will no longer be valid. Proper characterization of the form of the phase-space distribution is critical to analyzing kinetic effects such as particle trapping in intense laser-plasma interactions.

As well as modeling intense laser-plasma interactions, the relativistic, warm fluid theory of a nonequilibrium, collisionless plasma presented here can also be applied to model relativistic charged particle beams and beamplasma interactions, and may have applications to astrophysical plasmas.

\section{ACKNOWLEDGMENTS}

We would like to thank B. A. Shadwick for many valuable and insightful discussions on the topic of relativistic kinetic theories.

This work was supported by the Director, Office of Science, Office of High Energy Physics, of the U.S. Department of Energy under Contract No. DE-AC02$05 \mathrm{CH} 11231$.

\section{Appendix A: Relation between fluid quantities and warm fluid model}

In this appendix, the relativistic fluid model quantities of Sec. II are related to conventional fluid quantities (see, e.g., Ref. [1]). The energy density (in the local rest frame) may be defined as

$$
\mathrm{e}=U_{\mu} T^{\mu \nu} U_{\nu}
$$

where $U_{\mu}=J_{\mu} /\left(J_{\nu} J^{\nu}\right)^{1 / 2}=J_{\mu} / n_{p}$. Recall that $n_{p} U_{\mu}=$ $h u_{\mu}$ and $\left(n_{p} / h\right)^{2}=\gamma_{\text {th }}^{2}=1+\epsilon^{2}$. Using the centered moment definition Eq. (14) and the warm plasma assumption Eq. (23), the energy density in the warm fluid model is

$$
\mathrm{e}=h+h \epsilon^{2}
$$

where the invariant density $h$ can be identified as the enthalpy.

The pressure tensor may be defined as

$$
P^{\mu \nu}=\Delta_{\alpha}^{\mu} T^{\alpha \beta} \Delta_{\beta}^{\nu},
$$

where $\Delta^{\mu \nu}=g^{\mu \nu}-U^{\mu} U^{\nu}$ is the projector with the property $\Delta^{\mu \nu} U_{\nu}=0$. Using the centered moment definition Eq. (14), the pressure tensor is $P^{\mu \nu}=\Delta_{\alpha}^{\mu} \Theta^{\alpha \beta} \Delta_{\beta}^{\nu}$. In the warm plasma approximation $u_{\mu} \Theta^{\mu \nu}=0$, and the pressure tensor is equal to the momentum variance tensor $P^{\mu \nu}=\Theta^{\mu \nu}$. Since the plasma is assumed collisionless, the pressure is intrinsically anisotropic (in the local rest frame), and a local hydrostatic scalar pressure cannot describe the pressure tensor outside the $1 \mathrm{D}$ limit [28]. In 
the 1D limit, the contraction of the pressure tensor can be identified with the local hydrostatic scalar pressure $\mathrm{p}=-P_{\mu}^{\mu}=-\Theta_{\mu}^{\mu}=h \epsilon^{2}$.

The heat flow (difference of the energy flow and flow of enthalpy) may be defined as

$$
\mathrm{q}^{\mu}=U_{\nu} T^{\nu \alpha} \Delta_{\alpha}^{\mu}
$$

Evaluating the heat flow in terms of centered moments yields

$$
\begin{aligned}
\mathrm{q}^{\mu} & =\left(h / n_{p}\right)\left[U_{\sigma} Q_{\alpha}^{\alpha \sigma} U^{\mu}-Q_{\alpha}^{\mu \alpha}\right] / 2 \\
& =-\frac{1}{2} \frac{h}{n_{p}} Q_{\alpha}^{\alpha \mu}+\frac{1}{4}\left(\frac{h}{n_{p}}\right)^{2} U^{\mu}\left[\left(\Theta_{\nu}^{\nu}\right)^{2} / h-R_{\delta \alpha}^{\delta \alpha}\right],
\end{aligned}
$$

where $R^{\alpha \beta \mu \nu}=\int d \Omega f\left(p^{\alpha}-u^{\alpha}\right)\left(p^{\beta}-u^{\beta}\right)\left(p^{\mu}-u^{\mu}\right)\left(p^{\nu}-u^{\nu}\right)$ is the fourth-order centered moment. The heat flow is proportional to the third-order (and higher) moments, i.e., $\mathrm{q}^{\mu} \sim h \epsilon^{3}$. The heat flow is only proportional to third and higher-order moments because a collisionless plasma (i.e., no viscosity) was assumed, and $\mathrm{q}^{\mu}=0$ in the warm plasma model.

The Taub inequality [34]

$$
\mathrm{e} h \geq n_{p}^{2}
$$

can be expressed in terms of the centered moments as $\left(\Theta_{\nu}^{\nu}\right)^{2} \leq h R_{\alpha \delta}^{\alpha \delta}$, which is the Cauchy-Schwarz inequality for the moments of the distribution. Therefore, the Taub inequality must always be satisfied within the warm fluid model (to all orders in $\epsilon$ ). In the warm plasma approximation, assuming $\epsilon$ is a small parameter and expanding to $\mathcal{O}\left(\epsilon^{2}\right)$, eh $=n_{p}^{2}$. The warm plasma approximation is consistent with the Taub inequality.

\section{Appendix B: Invariant measure of thermal spread of a relativistic Maxwell distribution}

In this appendix, the invariant measure of thermal spread $\epsilon$ is calculated assuming a relativistic MaxwellBoltzmann (Jüttner) distribution. It is shown that the invariant measure of thermal spread is related to the width of the distribution.

Consider the distribution [1] given by

$$
f=f_{0} \exp \left[-p^{\mu} J_{\mu} /\left(n_{p} \theta_{\mathrm{J}}\right)\right]
$$

where $\theta_{\mathrm{J}}$ is the equilibrium temperature of the system (normalized to $m c^{2} / k_{\mathrm{B}}$ ) and $f_{0}$ is a constant. Note that, since $p^{\mu} J_{\mu}$ is a Lorentz scalar, $p^{\mu} J_{\mu}$ is invariant and may be evaluated in any frame. In the rest frame of the plasma fluid $J^{\mu}=n_{p}(1, \mathbf{0})$ and $p^{\mu} J_{\mu}=n_{p} \gamma$. In the rest frame of the plasma fluid, and assuming a warm plasma, $f=f_{0} \exp \left(-\gamma / \theta_{\mathrm{J}}\right)=f_{0} \exp \left[-\left(1-\beta^{2}\right)^{-1 / 2} / \theta_{\mathrm{J}}\right] \simeq$ $f_{1} \exp \left(-\beta^{2} / 2 \theta_{\mathrm{J}}\right)$, and the equilibrium temperature $\theta_{\mathrm{J}}$ is the root-mean square of the plasma velocity distribution.

In $1 \mathrm{D}$, the invariant momentum-space volume is $d \Omega=$ $d p / p^{0}=d p / \gamma=\left(\gamma^{2}-1\right)^{-1 / 2} d \gamma$. For a Jüttner distribution Eq. (B1) in 1D, the invariant density is

$$
h=\int d \Omega f=2 f_{0} \int_{1}^{\infty} d \gamma \frac{e^{-\gamma / \theta_{\mathrm{J}}}}{\left(\gamma^{2}-1\right)^{1 / 2}}=2 f_{0} K_{0}\left(\theta_{\mathrm{J}}^{-1}\right),
$$

and the proper density $n_{p}=\left(J_{\mu} J^{\mu}\right)^{1 / 2}$ is given by

$$
\begin{aligned}
n_{p} & =\frac{J_{\mu}}{n_{p}} \int d \Omega f p^{\mu}=2 f_{0} \int_{1}^{\infty} d \gamma \frac{\gamma e^{-\gamma / \theta_{\mathrm{J}}}}{\left(\gamma^{2}-1\right)^{1 / 2}} \\
& =2 f_{0} K_{1}\left(\theta_{\mathrm{J}}^{-1}\right),
\end{aligned}
$$

where $K_{\mathrm{m}}$ are $\mathrm{m}^{t h}$-order modified Bessel functions of the second kind. The ratio of proper to invariant densities is $\gamma_{\mathrm{th}}=n_{p} / h=K_{1}\left(\theta_{\mathrm{J}}^{-1}\right) / K_{0}\left(\theta_{\mathrm{J}}^{-1}\right)$, and the invariant measure of thermal spread is

$$
\epsilon^{2}=\left[K_{1}\left(\theta_{\mathrm{J}}^{-1}\right) / K_{0}\left(\theta_{\mathrm{J}}^{-1}\right)\right]^{2}-1 .
$$

Note, for large argument $\sigma \gg 1, K_{n}(\sigma) \simeq$ $\sqrt{(\pi / 2)} \sigma^{-1 / 2} e^{-\sigma}\left[1+\left(4 n^{2}-1\right) /(8 \sigma)\right]$. In the limit of nonrelativistic temperature $\theta_{\mathrm{J}} \ll 1$,

$$
\epsilon^{2} \simeq \frac{\left(1+3 \theta_{\mathrm{J}} / 8\right)^{2}}{\left(1-\theta_{\mathrm{J}} / 8\right)^{2}}-1 \simeq \theta_{\mathrm{J}}
$$

The invariant measure of thermal spread can be identified as the equilibrium temperature of a Jüttner (relativistic Maxwell-Boltzmann) distribution, $\epsilon^{2}=\theta_{\mathrm{J}}$.

In $3 \mathrm{D}$, the invariant momentum-space volume is $d \Omega=$ $d^{3} \boldsymbol{p} / p^{0}=4 \pi p^{2} d p / \gamma=4 \pi\left(\gamma^{2}-1\right)^{1 / 2} d \gamma$. For a Jüttner distribution Eq. (B1) in $3 \mathrm{D}$, the invariant density is

$$
h=4 \pi f_{0} \int_{1}^{\infty} d \gamma\left(\gamma^{2}-1\right)^{1 / 2} e^{-\gamma / \theta_{\mathrm{J}}}=4 \pi f_{0} \theta_{\mathrm{J}} K_{1}\left(\theta_{\mathrm{J}}^{-1}\right),
$$

and the proper density $n_{p}=\left(J_{\mu} J^{\mu}\right)^{1 / 2}$ is

$n_{p}=4 \pi f_{0} \int_{1}^{\infty} d \gamma\left(\gamma^{2}-1\right)^{1 / 2} \gamma e^{-\gamma / \theta_{\mathrm{J}}}=4 \pi f_{0} \theta_{\mathrm{J}} K_{2}\left(\theta_{\mathrm{J}}^{-1}\right)$.

The ratio of proper to invariant densities is $\gamma_{\mathrm{th}}=n_{p} / h=$ $K_{2}\left(\theta_{\mathrm{J}}^{-1}\right) / K_{1}\left(\theta_{\mathrm{J}}^{-1}\right)$, and the invariant measure of thermal spread is

$$
\epsilon^{2}=\left[K_{2}\left(\theta_{\mathrm{J}}^{-1}\right) / K_{1}\left(\theta_{\mathrm{J}}^{-1}\right)\right]^{2}-1 .
$$

In the limit of non-relativistic temperature $\theta_{\mathrm{J}} \ll 1$,

$$
\epsilon^{2} \simeq \frac{\left(1+15 \theta_{\mathrm{J}} / 8\right)^{2}}{\left(1+3 \theta_{\mathrm{J}} / 8\right)^{2}}-1 \simeq 3 \theta_{\mathrm{J}}
$$


[1] S. R. de Groot, W. A. van Leeuwen, and C. G. van Weert, Relativistic Kinetic Theory, North-Holland, Amsterdam, 1980.

[2] E. Esarey, C. B. Schroeder, and W. P. Leemans, Rev. Mod. Phys. 81, 1229 (2009).

[3] S. P. D. Mangles, C. D. Murphy, Z. Najmudin, A. G. R. Thomas, J. L. Collier, A. E. Dangor, E. J. Divall, P. S. Foster, J. G. Gallacher, C. J. Hooker, D. A. Jaroszynski, A. J. Langley, W. B. Mori, P. A. Norreys, F. S. Tsung, R. Viskup, B. R. Walton, and K. Krushelnick, Nature 431, 535 (2004).

[4] C. G. R. Geddes, C. Toth, J. van Tilborg, E. Esarey, C. B. Schroeder, D. Bruhwiler, C. Nieter, J. Cary, and W. P. Leemans, Nature 431, 538 (2004).

[5] J. Faure, Y. Glinec, A. Pukhov, S. Kiselev, S. Gordienko, E. Lefebvre, J.-P. Rousseau, F. Burgy, and V. Malka, Nature 431, 541 (2004).

[6] W. P. Leemans, B. Nagler, A. J. Gonsalves, C. Tóth, K. Nakamura, C. G. R. Geddes, E. Esarey, C. B. Schroeder, and S. M. Hooker, Nature Phys. 2, 696 (2006).

[7] C. B. Schroeder, E. Esarey, and B. A. Shadwick, Phys. Rev. E 72, 055401 (2005).

[8] A. I. Akhiezer and R. V. Polovin, Zh. Eksp. Teor. Fiz. 30, 915 (1956), [Sov. Phys. JETP 3, 696 (1956)].

[9] J. M. Dawson, Phys. Rev. 113, 383 (1959).

[10] T. P. Coffey, Phys. Fluids 14, 1402 (1971).

[11] P. Volfbeyn, E. Esarey, and W. Leemans, Phys. Plasmas 6, 2269 (1999).

[12] M. C. Marconi, C. H. Moreno, J. J. Rocca, V. N. Shlyaptsev, and A. L. Osterheld, Phys. Rev. E 62, 7209 (2000).

[13] J. B. Rosenzweig, Phys. Rev. A 38, 3634 (1988).

[14] T. Katsouleas and W. B. Mori, Phys. Rev. Lett. 61, 90 (1988).

[15] Z. M. Sheng and J. Meyer-ter-Vehn, Phys. Plasmas 4,
493 (1997).

[16] B. A. Shadwick, G. M. Tarkenton, and E. H. Esarey, Phys. Rev. Lett. 93, 175002 (2004).

[17] B. A. Shadwick, G. M. Tarkenton, E. H. Esarey, and C. B. Schroeder, Phys. Plasmas 12, 056710 (2005).

[18] J. Yazdanpanah, A. Anvari, and J. Samimi, Phys. Plasmas 16, 023104 (2009).

[19] D. R. Nicholson, Introduction to Plasma Theory, Krieger, 1992.

[20] J. D. Jackson, Classical Electrodynamics, Wiley, New York, 1975.

[21] W. A. Newcomb, Phys. Fluids 25, 846 (1982).

[22] P. Amendt, Phys. Fluids 29, 1458 (1986).

[23] J. G. Siambis, Phys. Fluids 30, 896 (1986).

[24] S. Pennisi and A. M. Anile, Phys. Fluids 3, 1091 (1991).

[25] C. Eckart, Phys. Rev. 58, 919 (1940).

[26] W. L. Kruer, The Physics of Laser Plasma Interactions, Addison-Wesley, Redwood City, CA, 1988.

[27] P. C. Clemmow and A. J. Wilson, Proc. R. Soc. London Ser. A 237, 117 (1956).

[28] W. A. Newcomb, Phys. Fluids 29, 1854 (1986).

[29] N. H. Matlis, S. Reed, S. S. Bulanov, V. Chvykov, G. Kalintchenko, T. Matsuoka, P. Rousseau, V. Yanovsky, A. Maksimchuk, S. Kalmykov, G. Shvets, and M. C. Downer, Nature Phys. 2, 749 (2006).

[30] C. B. Schroeder, E. Esarey, B. A. Shadwick, and W. P. Leemans, Phys. Plasmas 13, 033103 (2006).

[31] E. Esarey, C. B. Schroeder, E. Cormier-Michel, B. A. Shadwick, C. G. R. Geddes, and W. P. Leemans, Phys. Plasmas 14, 056708 (2007).

[32] J. Krall, G. Joyce, and E. Esarey, Phys. Rev. A 44, 6854 (1991).

[33] D. A. Burton and A. Noble, J. Phys. A: Math. Theor. 43, 075502 (2010).

[34] A. H. Taub, Phys. Rev. 74, 328 (1948). 